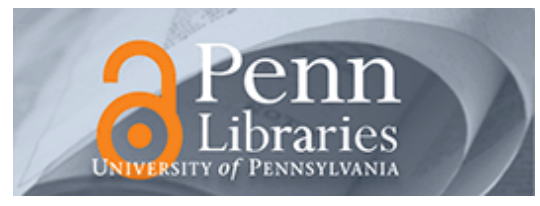

University of Pennsylvania

ScholarlyCommons

$12-22-2009$

\title{
Galaxy-CMB and Galaxy-Galaxy Lensing on Large Scales: Sensitivity to Primordial Non-Gaussianity
}

\author{
Donghui Jeong \\ University of Texas at Austin \\ Eiichiro Komatsu \\ University of Texas at Austin \\ Bhuvnesh Jain \\ University of Pennsylvania, bjain@physics.upenn.edu
}

Follow this and additional works at: https://repository.upenn.edu/physics_papers

Part of the Physics Commons

\section{Recommended Citation}

Jeong, D., Komatsu, E., \& Jain, B. (2009). Galaxy-CMB and Galaxy-Galaxy Lensing on Large Scales:

Sensitivity to Primordial Non-Gaussianity. Retrieved from https://repository.upenn.edu/physics_papers/44

\footnotetext{
Suggested Citation:

Jeong, D., E. Komatsu and B. Jain. (2009). "Galaxy-CMB and galaxy-galaxy lensing on large scales: Sensitivity to primordial non-Guassianity." Physical Review D. 80, 123527.

(c) 2009 The American Physical Review.

http://dx.doi.org/10.1103/PhysRevD.80.123527

This paper is posted at ScholarlyCommons. https://repository.upenn.edu/physics_papers/44

For more information, please contact repository@pobox.upenn.edu.
} 


\title{
Galaxy-CMB and Galaxy-Galaxy Lensing on Large Scales: Sensitivity to Primordial Non-Gaussianity
}

\author{
Abstract \\ A convincing detection of primordial non-Gaussianity in the local form of the bispectrum, whose \\ amplitude is given by the $f_{N L}$ parameter, offers a powerful test of inflation. In this paper, we calculate the \\ modification of two-point cross-correlation statistics of weak lensing-galaxy-galaxy lensing and \\ galaxycosmic microwave background (CMB) crosscorrelation-due to $f_{N L}$. We derive and calculate the \\ covariance matrix of galaxy-galaxy lensing, including cosmic variance terms. We focus on large scales $(I<$ \\ 100) for which the shape noise of the shear measurement becomes irrelevant and cosmic variance \\ dominates the error budget. For a modest degree of non-Gaussianity, $f_{\mathrm{NL}}= \pm 50$ modifications of the \\ galaxy-galaxy-lensing signal at the $10 \%$ level are seen on scales $\mathrm{R} \sim 300 \mathrm{Mpc}$, and grow rapidly toward \\ larger scales as $\propto R^{2}$. We also see a clear signature of the baryonic acoustic oscillation feature in the \\ matter power spectrum at $\sim 150 \mathrm{Mpc}$, which can be measured by next-generation lensing experiments. In \\ addition, we can probe the local-form primordial non-Gaussianity in the galaxy-CMB lensing signal by \\ correlating the lensing potential reconstructed from $\mathrm{CMB}$ with high- $z$ galaxies. For example, for $\mathrm{f}_{\mathrm{NL}}= \pm 50$, \\ we find that the galaxy-CMB lensing cross-power spectrum is modified by $\sim 10 \%$ at $I \sim 40$, and by a factor \\ of 2 at $I \sim 10$, for a population of galaxies at $z=2$ with a bias of 2 . The effect is greater for more highly \\ biased populations at larger $z$, thus, high-z galaxy surveys cross correlated with CMB offer a yet another \\ probe of primordial non-Gaussianity. \\ Disciplines \\ Physical Sciences and Mathematics | Physics
}

\section{Comments}

Suggested Citation:

Jeong, D., E. Komatsu and B. Jain. (2009). "Galaxy-CMB and galaxy-galaxy lensing on large scales:

Sensitivity to primordial non-Guassianity." Physical Review D. 80, 123527.

(C) 2009 The American Physical Review.

http://dx.doi.org/10.1103/PhysRevD.80.123527 
PHYSICAL REVIEW D 80, 123527 (2009)

\title{
Galaxy-CMB and galaxy-galaxy lensing on large scales: Sensitivity to primordial non-Gaussianity
}

\author{
Donghui Jeong* and Eiichiro Komatsu \\ Texas Cosmology Center and Department of Astronomy, University of Texas at Austin, 1 University Station, C1400, \\ Austin, Texas 78712, USA
}

Bhuvnesh Jain

Particle Cosmology Center and Department of Physics and Astronomy, University of Pennsylvania, Philadelphia, Pennsylvania 19104, USA

(Received 16 October 2009; published 22 December 2009)

\begin{abstract}
A convincing detection of primordial non-Gaussianity in the local form of the bispectrum, whose amplitude is given by the $f_{\mathrm{NL}}$ parameter, offers a powerful test of inflation. In this paper, we calculate the modification of two-point cross-correlation statistics of weak lensing - galaxy-galaxy lensing and galaxycosmic microwave background (CMB) crosscorrelation-due to $f_{\mathrm{NL}}$. We derive and calculate the covariance matrix of galaxy-galaxy lensing, including cosmic variance terms. We focus on large scales $(l<100)$ for which the shape noise of the shear measurement becomes irrelevant and cosmic variance dominates the error budget. For a modest degree of non-Gaussianity, $f_{\mathrm{NL}}= \pm 50$ modifications of the galaxy-galaxy-lensing signal at the $10 \%$ level are seen on scales $R \sim 300 \mathrm{Mpc}$, and grow rapidly toward larger scales as $\propto R^{2}$. We also see a clear signature of the baryonic acoustic oscillation feature in the matter power spectrum at $\sim 150 \mathrm{Mpc}$, which can be measured by next-generation lensing experiments. In addition, we can probe the local-form primordial non-Gaussianity in the galaxy-CMB lensing signal by correlating the lensing potential reconstructed from $\mathrm{CMB}$ with high- $z$ galaxies. For example, for $f_{\mathrm{NL}}=$ \pm 50 , we find that the galaxy-CMB lensing cross-power spectrum is modified by $\sim 10 \%$ at $l \sim 40$, and by a factor of 2 at $l \sim 10$, for a population of galaxies at $z=2$ with a bias of 2 . The effect is greater for more highly biased populations at larger $z$; thus, high- $z$ galaxy surveys cross correlated with CMB offer a yet another probe of primordial non-Gaussianity.
\end{abstract}

DOI: 10.1103/PhysRevD.80.123527

PACS numbers: 98.62.Sb, 98.65.- r, 98.80.-k

\section{INTRODUCTION}

Why study non-Gaussianity? For many years it was recognized that the simple inflationary models based upon a single slowly-rolling scalar field would predict nearly Gaussian primordial fluctuations. In particular, when we parametrize the magnitude of non-Gaussianity in the primordial curvature perturbations $\zeta$, which gives the observed temperature anisotropy in the cosmic microwave Bbackground (CMB) in the Sachs-Wolfe limit as $\Delta T / T=$ $-\zeta / 5$, using the so-called nonlinear parameter $f_{\mathrm{NL}}[1]$ as $\zeta(\mathbf{x})=\zeta_{L}(\mathbf{x})+\left(3 f_{\mathrm{NL}} / 5\right) \zeta_{L}^{2}(\mathbf{x})$, then the bispectrum of $\zeta$ is given by ${ }^{1} B_{\zeta}\left(k_{1}, k_{2}, k_{3}\right)=\left(6 f_{\mathrm{NL}} / 5\right)\left[P_{\zeta}\left(k_{1}\right) P_{\zeta}\left(k_{2}\right)+\right.$ (2 cyclic terms)], where $P_{\zeta}(k) \propto k^{n_{s}-4}$ is the power spectrum of $\zeta$ and $n_{s}$ is the tilt of the power spectrum, constrained as $n_{s}=0.960 \pm 0.013$ by the Wilkinson microwave anisotropy probe (WMAP) 5-year data [2]. This form of the bispectrum has the maximum signal in the so-called squeezed triangle for which $k_{3} \ll k_{2} \approx k_{1}$ [3]. In this limit, we obtain

\footnotetext{
*djeong@astro.as.utexas.edu

${ }^{1}$ Definition of the bispectrum in terms of Fourier coefficients of $\zeta$ is $\left\langle\zeta_{\mathbf{k}_{1}} \zeta_{\mathbf{k}_{2}} \zeta_{\mathbf{k}_{3}}\right\rangle=(2 \pi)^{3} \delta\left(\mathbf{k}_{1}+\mathbf{k}_{2}+\mathbf{k}_{3}\right) B_{\zeta}\left(k_{1}, k_{2}, k_{3}\right)$. Throughout this paper we shall order $k_{i}$ such that $k_{3} \leq k_{2} \leq k_{1}$.
}

$$
B_{\zeta}\left(k_{1}, k_{1}, k_{3} \rightarrow 0\right)=\frac{12}{5} f_{\mathrm{NL}} P_{\zeta}\left(k_{1}\right) P_{\zeta}\left(k_{3}\right) .
$$

The earlier calculations showed that $f_{\mathrm{NL}}$ from single-field slow-roll inflation would be of order the slow-roll parameter, $\epsilon \sim 10^{-2}[4-6]$. However, it is not until recent that it is finally realized that the coefficient of $P_{\zeta}\left(k_{1}\right) P_{\zeta}\left(k_{3}\right)$ from the simplest single-field slow-roll inflation with the canonical kinetic term in the squeezed limit is given precisely by $[7,8]$

$$
B_{\zeta}\left(k_{1}, k_{1}, k_{3} \rightarrow 0\right)=\left(1-n_{s}\right) P_{\zeta}\left(k_{1}\right) P_{\zeta}\left(k_{3}\right) .
$$

Comparing this result with the form predicted by the $f_{\mathrm{NL}}$ model, one obtains $f_{\mathrm{NL}}=(5 / 12)\left(1-n_{s}\right)$.

Perhaps, the most important theoretical discovery regarding primordial non-Gaussianity from inflation over the last few years is that, not only models with the canonical kinetic term, but all single-inflation models predict the bispectrum in the squeezed limit given by Eq. (2), regardless of the form of potential, kinetic term, slow-roll, or initial vacuum state [9-12]. Therefore, the prediction from all single-field inflation models is $f_{\mathrm{NL}}=(5 / 12)\left(1-n_{s}\right)=$ 0.017 for $n_{s}=0.96$. A convincing detection of $f_{\mathrm{NL}}$ well above this level is a breakthrough in our understanding of 
the physics of very early universe [13,14]. The current limit from the WMAP 5-year data is $f_{\mathrm{NL}}=38 \pm 21(68 \% \mathrm{CL})$ [15].

There are many ways of measuring $f_{\mathrm{NL}}$. The most popular method has been the bispectrum of CMB [1,1619] (also see [20] for a pedagogical review). The other methods include the trispectrum of CMB [21,22], the bispectrum of galaxies [23-26], and the abundance of galaxies and clusters of galaxies [27-30].

Recently, analytical [31-35] and numerical [31,36-38] studies of the effects of primordial non-Gaussianity on the power spectrum of dark matter halos $P_{h}(k)$ have revealed an unexpected signature of primordial non-Gaussianity in the form of a scale-dependent galaxy bias, i.e., $P_{h}(k)=$ $b_{1}^{2} P_{m}(k) \rightarrow\left[b_{1}+\Delta b(k)\right]^{2} P_{m}(k)$, where $P_{m}(k)$ is the power spectrum of matter density fluctuations, and

$$
\Delta b(k)=\frac{3\left(b_{1}-1\right) f_{\mathrm{NL}} \Omega_{m} H_{0}^{2} \delta_{c}}{D(z) k^{2} T(k)} .
$$

Here, $D(z)$ and $T(k)$ are the growth rate and the transfer function for linear matter density fluctuations, respectively, and $\delta_{c}=1.68$ is the threshold linear density contrast for a spherical collapse of an overdensity region. The $k^{2}$ factor in the denominator of $\Delta b(k)$ shows that this effect is important only on very large scales. Highly biased tracers are more sensitive to $f_{\mathrm{NL}}$.

\section{HALO-MASS CORRELATION FROM GALAXY- GALAXY LENSING}

\section{A. Formula}

The scale-dependent bias was theoretically discovered when the authors of [31] studied the form of the crosscorrelation power spectrum between the dark matter halos and the underlying matter density fluctuations, $P_{h m}(k)=$ $\left[b_{1}+\Delta b(k)\right] P_{m}(k)$. We can observe $P_{h m}(k)$ by cross correlating the locations of galaxies or clusters of galaxies with the matter density fluctuations traced by the weak gravitational lensing (see [39] for a review).

One efficient way of measuring $P_{h m}(k)$ is to use the socalled galaxy-galaxy-lensing technique [40-46]: choose one lens galaxy at a redshift $z_{L}$, and measure the mean of tangential shears in images of lensed (source or background) galaxies around the chosen central lensing galaxy as a function of radii from that central galaxy. Finally, average those mean tangential shears over all lensing galaxies at the same redshift $z_{L}$.

We begin with the definition of the tangential shear $\gamma_{t}$ on the flat $s k y^{2}$

$$
\gamma_{t}(\boldsymbol{\theta})=-\gamma_{1}(\boldsymbol{\theta}) \cos (2 \phi)-\gamma_{2}(\boldsymbol{\theta}) \sin (2 \phi),
$$

where $\boldsymbol{\theta}=(\theta \cos \phi, \theta \sin \phi)$, and $\gamma_{1}$ and $\gamma_{2}$ are compo-

\footnotetext{
${ }^{2}$ For an all-sky analysis, this relation needs to be replaced with the exact relation using the spin-2 harmonics [47].
}

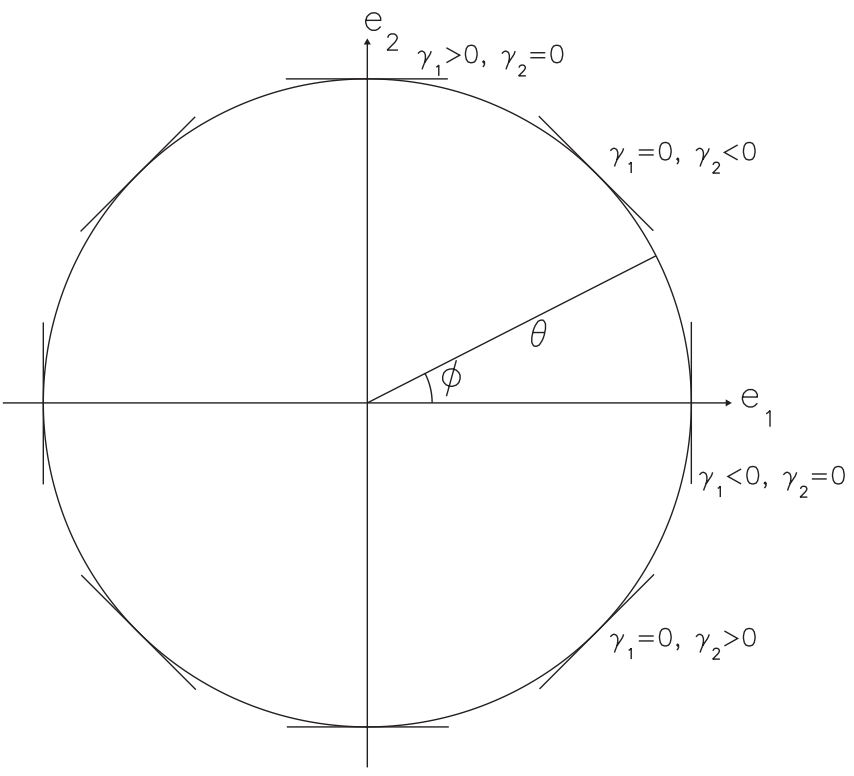

FIG. 1. Coordinate system and $\gamma_{1}$ and $\gamma_{2}$. The shear along $\boldsymbol{e}_{1}$ has $\gamma_{1}>0$ and $\gamma_{2}=0$, whereas the shear along $\boldsymbol{e}_{2}$ has $\gamma_{1}<0$ and $\gamma_{2}=0$. The shear along $\boldsymbol{e}_{1}+\boldsymbol{e}_{2}$ has $\gamma_{1}=0$ and $\gamma_{2}>0$, whereas the shear along $\boldsymbol{e}_{1}-\boldsymbol{e}_{2}$ has $\gamma_{1}=0$ and $\gamma_{2}<0$.

nents of the shear field. ${ }^{3}$ The coordinate system and the meaning of $\gamma_{1}$ and $\gamma_{2}$ are explained in Fig. 1. For purely tangential shears shown in Fig. 1, $\gamma_{t}$ is always positive. This property allows us to average $\gamma_{t}$ over the ring around the origin to estimate the mean tangential shear $\bar{\gamma}_{t}$ :

$$
\bar{\gamma}_{t}(\theta) \equiv \int_{0}^{2 \pi} \frac{d \phi}{2 \pi} \gamma_{t}(\theta, \phi) .
$$

On the flat sky, $\gamma_{1}$ and $\gamma_{2}$ are related to the projected massdensity fluctuation in Fourier space $\kappa(\boldsymbol{l})$ as

$$
\begin{aligned}
& \gamma_{1}(\boldsymbol{\theta})=\int \frac{d^{2} \boldsymbol{l}}{(2 \pi)^{2}} \kappa(\boldsymbol{l}) \cos (2 \varphi) e^{i \boldsymbol{l} \cdot \boldsymbol{\theta}}, \\
& \gamma_{2}(\boldsymbol{\theta})=\int \frac{d^{2} \boldsymbol{l}}{(2 \pi)^{2}} \kappa(\boldsymbol{l}) \sin (2 \varphi) e^{i \boldsymbol{l} \cdot \boldsymbol{\theta}},
\end{aligned}
$$

where $\varphi$ is the angle between $\boldsymbol{l}$ and $\boldsymbol{e}_{1}$, i.e., $\boldsymbol{l}=$ $(l \cos \varphi, l \sin \varphi)$. Using Eqs. (6) and (7) in Eq. (4), we write the tangential shear in terms of $\kappa(l)$ as

$$
\gamma_{t}(\boldsymbol{\theta})=-\int \frac{d^{2} \boldsymbol{l}}{(2 \pi)^{2}} \kappa(\boldsymbol{l}) \cos [2(\phi-\varphi)] e^{i l \theta \cos (\phi-\varphi)} .
$$

\footnotetext{
${ }^{3}$ As the shear has two independent components, we are ignoring another linear combination of $\gamma_{1}$ and $\gamma_{2}$ by only focusing on the tangential shear. In particular, on large scales there is information in the other component of the shear, and thus the full analysis including both shear components (not just tangential one) yields a modest (smaller than a factor of $\sqrt{2}$ ) improvement in the signal-to-noise ratio. Moreover, using magnification (in addition to shears), which is proportional to the convergence field $\kappa$, can also yield a modest improvement.
} 
The mean tangential shear [Eq. (5)] is then given by

$$
\begin{aligned}
\bar{\gamma}_{t}(\theta)= & -\int \frac{d^{2} \boldsymbol{l}}{(2 \pi)^{2}} \kappa(\boldsymbol{l}) \int_{0}^{2 \pi} \frac{d \boldsymbol{\phi}}{2 \pi} \cos [2(\phi-\varphi)] \\
& \times e^{i l \theta \cos (\phi-\varphi)} \\
= & \int \frac{d^{2} \boldsymbol{l}}{(2 \pi)^{2}} \kappa(\boldsymbol{l}) J_{2}(l \theta) .
\end{aligned}
$$

Here, we have used the identity

$$
J_{m}(x)=\int_{\alpha}^{2 \pi+\alpha} \frac{d \psi}{2 \pi} e^{i(m \psi-x \sin \psi)},
$$

with $m=2, \quad \psi=\phi-\varphi-\pi / 2, \quad \alpha=\varphi+\pi / 2$, and $\int_{0}^{2 \pi} d \psi \sin (2 \psi) e^{i x \cos \psi}=0$.

The ensemble average of the mean tangential shear vanishes, i.e., $\left\langle\bar{\gamma}_{t}\right\rangle=0$, as $\langle\kappa\rangle=0$. This simply means that the average of the mean tangential shears, measured with respect to random points on the sky, vanishes. We obtain nonzero values when we average the mean tangential shears measured with respect to the locations of halos (galaxies or clusters of galaxies). This quantity, called the galaxy-galaxy lensing or cluster-galaxy lensing, can be used to measure the halo-mass cross correlation.

While clusters of galaxies may be identified directly with dark matter halos of a given mass, how are galaxies related to halos? Some galaxies ("field galaxies") may also be identified directly with dark matter halos; however, galaxies residing within groups or clusters of galaxies should be identified with subhalos moving in a bigger dark matter halo. For such subhalos, our argument given below may not be immediately used. However, it is observationally feasible to identify the central galaxies in groups or clusters of galaxies and measure the mean tangential shear around them. A number of studies of luminous red galaxies (LRGs) extracted from the Sloan Digital Sky Survey (SDSS) have shown that these are typical central galaxies in galaxy groups $[46,48,49]$. Scalings such as the mass-luminosity scaling imply that LRGs provide a useful proxy for the halos within which they reside. We will assume in this study that such tracers will enable the halo-shear cross correlation to be measured. There are some caveats such as bimodal mass distributions in galaxy groups [49] and the extrapolation to higher redshift, but we will leave a detailed exploration to real galaxy tracers for later work.

The ensemble average of the mean tangential shears relative to the locations of halos at a given redshift $z_{L}$, denoted as $\left\langle\bar{\gamma}_{t}^{h}\right\rangle\left(\theta, z_{L}\right)$, is related to the angular crosscorrelation power spectrum of halos and $\kappa, C_{l}^{h \kappa}$, as [50]

$$
\left\langle\bar{\gamma}_{t}^{h}\right\rangle\left(\theta, z_{L}\right)=\int \frac{l d l}{2 \pi} C_{l}^{h \kappa}\left(z_{L}\right) J_{2}(l \theta) .
$$

We give the derivation of this result in Appendix A.

With the lens redshift $z_{L}$ known (from spectroscopic observations), we can calculate the comoving radius, $R$, corresponding to the angular separation on the sky $\theta$ as $R=\theta d_{A}\left(0 ; z_{L}\right)$ where $d_{A}\left(0 ; z_{L}\right)$ is the comoving angular diameter distance from $z=0$ to $z=z_{L}$. Using Limber's approximation [51,52] on the flat sky relating $C_{l}^{h \kappa}$ to $P_{h m}(k),{ }^{4}$ we can write Eq. (11) as [50]

$$
\left\langle\bar{\gamma}_{t}^{h}\right\rangle\left(R, z_{L}\right)=\frac{\rho_{0}}{\Sigma_{c}\left(z_{L}\right)} \int \frac{k d k}{2 \pi} P_{h m}\left(k, z_{L}\right) J_{2}(k R) .
$$

Here, $\rho_{0}$ is the mean comoving mass density of the Universe, and $\Sigma_{c}\left(z_{L}\right)$ is the so-called critical surface density:

$\Sigma_{c}^{-1}\left(z_{L}\right)=\frac{4 \pi G}{c^{2}}\left(1+z_{L}\right) d_{A}\left(0 ; z_{L}\right) \int_{z_{L}}^{\infty} d z_{S} p\left(z_{S}\right) \frac{d_{A}\left(z_{L} ; z_{S}\right)}{d_{A}\left(0 ; z_{S}\right)}$,

where $p\left(z_{S}\right)$ is the redshift distribution of sources normalized to unity $\int d z p(z)=1$ and $d_{A}(0 ; z)$ and $d_{A}\left(z ; z_{S}\right)$ are the comoving angular diameter distances out to $z$ and between $z$ and $z_{S}$, respectively. The numerical value of $4 \pi G / c^{2}$ is $6.01 \times 10^{-19} \mathrm{Mpc} / \mathrm{M}_{\odot}$, and $4 \pi G \rho_{0} / c^{2}$ is $1.67 \times 10^{-7}\left(\Omega_{m} h^{2}\right) \mathrm{Mpc}^{-2}$.

Equation (12) is often written as

$$
\left\langle\bar{\gamma}_{t}^{h}\right\rangle\left(R, z_{L}\right)=\frac{\Delta \Sigma\left(R, z_{L}\right)}{\Sigma_{c}\left(z_{L}\right)} .
$$

To simplify the analysis, let us define the "effective source redshift" of a given survey from the following equation:

$$
\frac{d_{A}\left(z_{L} ; z_{S, \mathrm{eff}}\right)}{d_{A}\left(0 ; z_{S, \mathrm{eff}}\right)} \equiv \int_{z_{L}}^{\infty} d z_{S} p\left(z_{S}\right) \frac{d_{A}\left(z_{L} ; z_{S}\right)}{d_{A}\left(0 ; z_{S}\right)}
$$

Henceforth, we shall use $z_{S}$ to denote $z_{S \text {,eff }}$, and write

$$
\Sigma_{c}^{-1}\left(z_{L} ; z_{S}\right)=\frac{4 \pi G}{c^{2}}\left(1+z_{L}\right) d_{A}\left(0 ; z_{L}\right) \frac{d_{A}\left(z_{L} ; z_{S}\right)}{d_{A}\left(0 ; z_{S}\right)} .
$$

Figure 2 shows $\Sigma_{c}$ for $z_{L}=0.1$ (2dFGRS, Two Degree Field Galaxy Redshift Survey), 0.2 (SDSS main), 0.3 (SDSS LRG), and 0.5 and 0.8 (both LSST, Large Synoptic Survey Telescope). The smaller $\Sigma_{c}$ is, the larger the observed mean tangential shear is.

\section{B. Results}

We can now calculate the observable $\Delta \Sigma\left(R, z_{L}\right)$ for various values of $f_{\mathrm{NL}}$. We use

\footnotetext{
${ }^{4}$ As we are dealing with correlations on very large angular scales, one may worry about the validity of Limber's approximation. In Appendix C, we give a detailed study of the validity and limitation of Limber's approximation for the galaxy-galaxy lensing.
} 


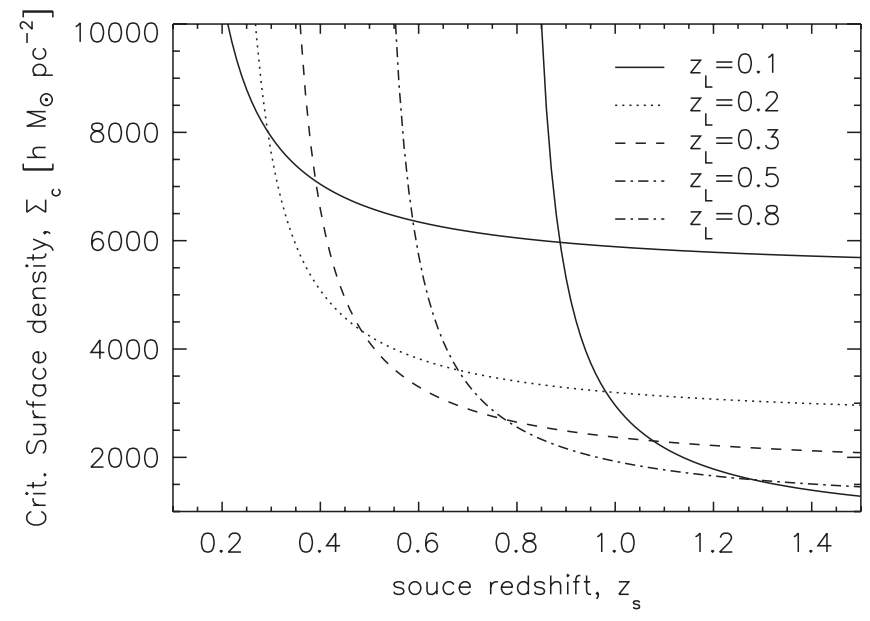

FIG. 2. Critical surface density $\Sigma_{c}\left(z_{L} ; z_{S}\right)$ as a function of the source redshift $z_{S}$ for various lens redshifts that roughly correspond to the Two Degree Field Galaxy Redshift Survey (2dFGRS; $z_{L}=0.1$, solid), the main sample of the Sloan Digital Sky Survey (SDSS; $z_{L}=0.2$, dotted), the luminous red galaxies (LRGs) of SDSS $\left(z_{L}=0.3\right.$, dashed), and the Large Synoptic Survey Telescope (LSST; $z_{L}=0.5$ and 0.8, dot-dashed and triple-dot-dashed, respectively).

$$
\begin{aligned}
\Delta \Sigma\left(R, z_{L}\right)= & \rho_{0} b_{1} \int \frac{k d k}{2 \pi} P_{m}\left(k, z_{L}\right) J_{2}(k R) \\
& +\rho_{0} \int \frac{k d k}{2 \pi} \Delta b\left(k, z_{L}\right) P_{m}\left(k, z_{L}\right) J_{2}(k R)
\end{aligned}
$$

where the scale-dependent bias $\Delta b(k, z)$ is given by Eq. (3). As we are interested in large scales, i.e., $R>$ $10 h^{-1} \mathrm{Mpc}$, we shall use the linear matter spectrum for $P_{m}(k)$.

Figure 3 shows, for the Gaussian initial condition $\left(f_{\mathrm{NL}}=0\right), \Delta \Sigma\left(R, z_{L}\right)$ from $R=50$ to $200 h^{-1}$ Mpc. We have chosen the bias parameters and lens redshifts to represent the existing data sets as well as the future ones: $b_{1}=2$ at $z_{L}=0.3$ (similar to the observed values from SDSS LRGs [53], solid line), $b_{1}=2$ at $z_{L}=0.5$ (higher- $z$ LRGs [54], dotted line), $b_{1}=2$ at $z_{L}=0.8$ (galaxies that can be observed by LSST, [55], dashed line), and $b_{1}=5$ at $z_{L}=0.8$ (clusters of galaxies that can be observed by LSST, dot-dashed line). While LSST is an imaging survey, we assume that we can obtain spectroscopic redshifts of some $\left(\sim 10^{6}\right)$ lens galaxies by follow-up observations. It is also straightforward to extend our analysis to lenses selected by photometric redshifts.

At $R \sim 110 h^{-1} \mathrm{Mpc}$, we see a clear "shoulder" due to the baryonic feature in the linear matter power spectrum (often called baryon acoustic oscillations; BAO). The sound horizon at the drag epoch (which is more relevant to the matter power spectrum than the photon decoupling epoch for the CMB power spectrum) calculated from the cosmological model that we use, the "WMAP + BAO +

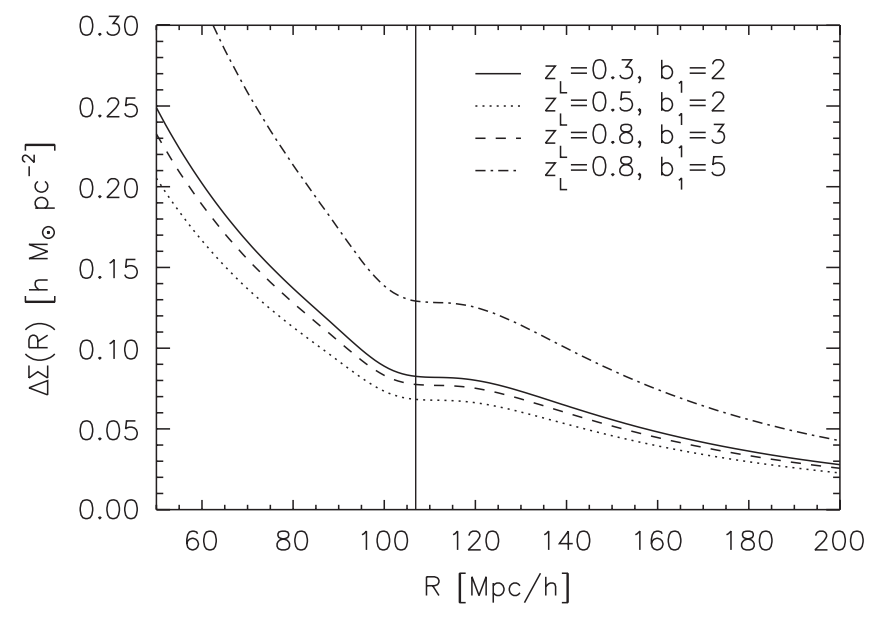

FIG. 3. The baryonic feature in the matter power spectrum, as seen in the galaxy-galaxy lensing $\Delta \Sigma(R)$ for several populations of lens galaxies with $b_{1}=2$ at $z_{L}=0.3$ (similar to SDSS LRGs, solid), $b_{1}=2$ at $z_{L}=0.5$ (higher- $z$ LRGs, dotted), $b_{1}=2$ at $z_{L}=0.8$ (galaxies that can be observed by LSST, dashed), and $b_{1}=5$ at $z_{L}=0.8$ (clusters of galaxies that can be observed by LSST, dot-dashed). The vertical line shows the location of the baryonic feature, $R_{\mathrm{BAO}}=106.9 \mathrm{~h}^{-1} \mathrm{Mpc}$, calculated from the "WMAP + BAO + SN ML" parameters in Table 1 of [2]. Note that we have used the linear matter power spectrum and the Gaussian initial condition $\left(f_{\mathrm{NL}}=0\right)$ for this calculation.

SN ML" (where SN stands for supernova and ML represents maximum likelihood) parameters in Table 1 of [2], is $106.9 h^{-1} \mathrm{Mpc}$, as shown as the vertical line in this figure. The magnitude of $\Delta \Sigma$ on this scale is $\sim 0.1 h \mathrm{M}_{\odot} \mathrm{pc}^{-2}$. Assuming a range of $\Sigma_{c}$ from future surveys $\Sigma_{c} \sim$ $1000-4000 h \mathrm{M}_{\odot} \mathrm{pc}^{-2}$ (see Fig. 2), this value corresponds to the mean tangential shear of order $2.5 \times 10^{-5}$ to $10^{-4}$. Is this observable?

For comparison, Sheldon et al. [48] measured $\Delta \Sigma(R) \sim$ $0.5 h \mathrm{M}_{\odot} \mathrm{pc}^{-2}$ at $R \sim 30 h^{-1} \mathrm{Mpc}$ from clusters of galaxies in the SDSS main sample. The mean lens redshift for these data is $z_{L} \sim 0.2$, which would give $\Sigma_{c} \sim 5000 h \mathrm{M}_{\odot} \mathrm{pc}^{-2}$ (see Fig. 2 for $z_{L}=0.2$ and $z_{S} \sim 0.4$ ); thus, the magnitude of the mean tangential shear that they were able to measure is of order $10^{-4}$, which is only $\sim 1$ to 4 times larger than the magnitude of the signal expected from the BAO. Therefore, detecting the BAO signature in $\Delta \Sigma(R)$ should be quite feasible with the future observations. We shall give a more quantitative discussion on the detectability of BAO from the galaxy-galaxy-lensing effect in Sec IID.

How about $f_{\mathrm{NL}}$ ? As expected, the effect of $f_{\mathrm{NL}}$ is enhanced on very large scales, i.e., hundreds of Mpc (see Fig. 4). For $f_{\mathrm{NL}}= \pm 50, \Delta \Sigma(R)$ is modified by $10-20 \%$ at $R \sim 300 h^{-1}$ Mpc (depending on $b_{1}$ and $z_{L}$; see Fig. 5). The modification grows rapidly toward larger scales, in proportion to $R^{2}$. On such a large scale $\left(R \sim 300 h^{-1} \mathrm{Mpc}\right)$, the galaxy-galaxy-lensing signal is on the order of $\Delta \Sigma \sim 0.01 h \mathrm{M}_{\odot} \mathrm{pc}^{-2}$, and thus we need to measure the mean tangential shear down to the level of $\bar{\gamma}_{t}^{h} \sim 2.5 \times$ $10^{-6}$ to $10^{-5}$, i.e., $10-40$ times smaller than the level of 


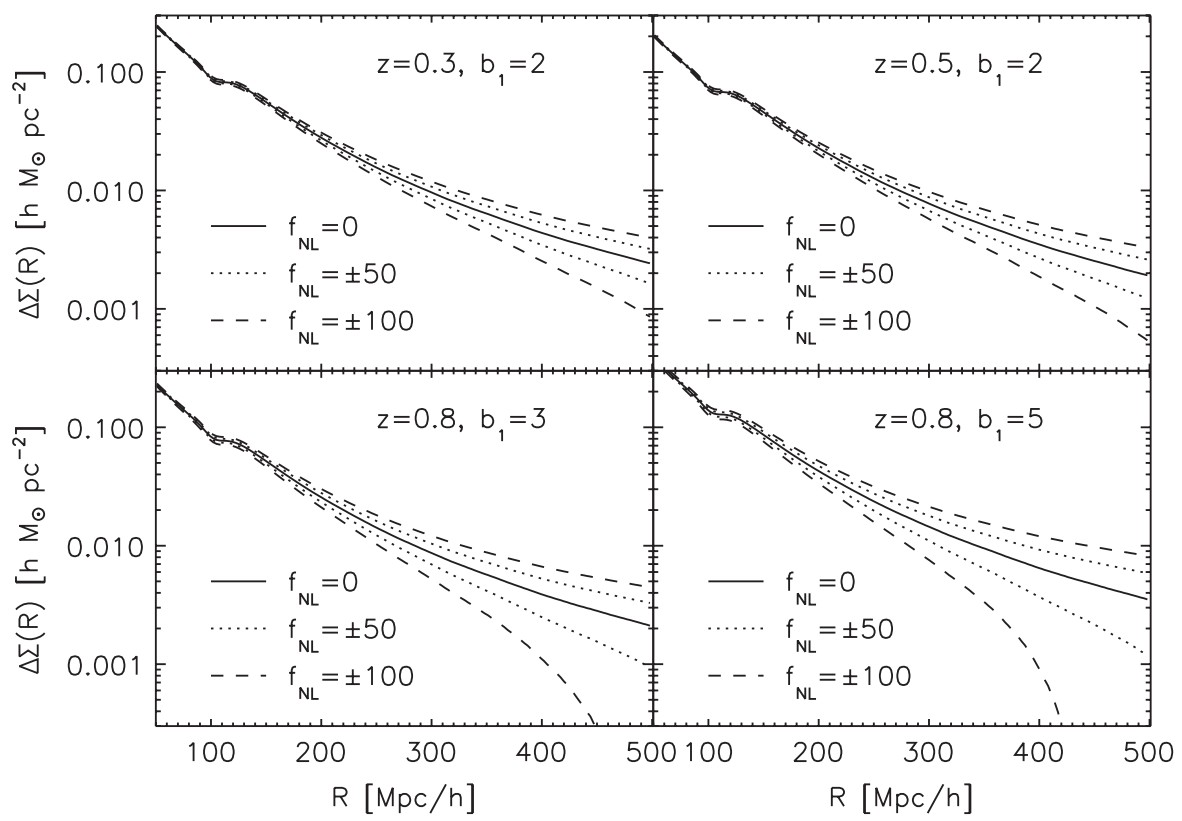

FIG. 4. Imprints of the local-type primordial non-Gaussianity in the galaxy-galaxy lensing $\Delta \Sigma(R)$ for the same populations of lens galaxies as in Fig. 3. The solid, dashed, and dotted lines show $f_{\mathrm{NL}}=0, \pm 50$, and \pm 100 , respectively.

sensitivity achieved by the current observations. Can we the mean tangential shear is

observe such a small shear?

\section{Covariance matrix of the mean tangential shear}

In order to study the feasibility of measuring the tangential shear of order $10^{-6}$, we compute the covariance matrix of the mean tangential shears averaged over $N_{L}$ lens galaxies. As derived in Appendix B, the covariance matrix of

$$
\begin{aligned}
& \left\langle\bar{\gamma}_{t}^{h}(\theta) \bar{\gamma}_{t}^{h}\left(\theta^{\prime}\right)\right\rangle-\left\langle\bar{\gamma}_{t}^{h}(\theta)\right\rangle\left\langle\bar{\gamma}_{t}^{h}\left(\theta^{\prime}\right)\right\rangle \\
& =\frac{1}{4 \pi f_{\text {sky }}} \int \frac{l d l}{2 \pi} J_{2}(l \theta) J_{2}\left(l \theta^{\prime}\right) \\
& \quad \times\left[\left(C_{l}^{h \kappa}\right)^{2}+\left(C_{l}^{h}+\frac{1}{n_{L}}\right)\left(C_{l}^{\kappa}+\frac{\sigma_{\gamma}^{2}}{n_{S}}\right)\right] .
\end{aligned}
$$

This expression includes the cosmic variance, the shot

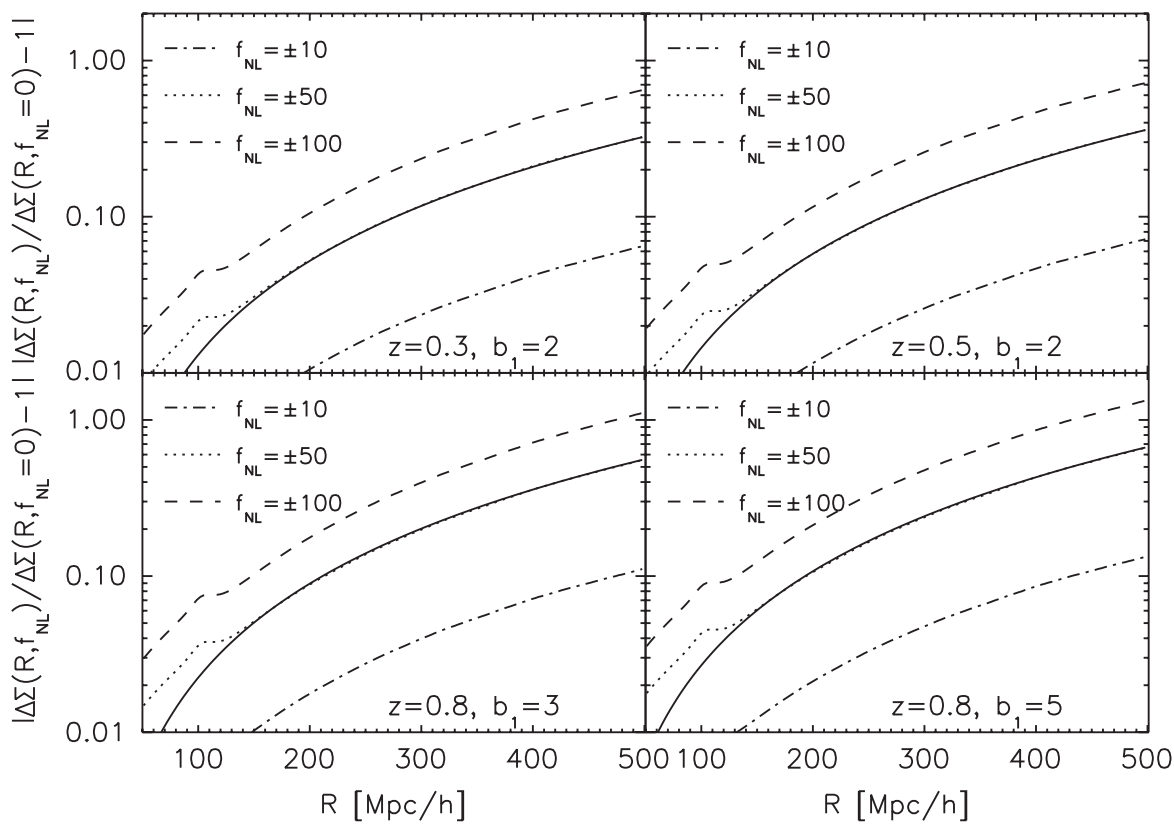

FIG. 5. Fractional differences between $\Delta \Sigma(R)$ from non-Gaussian initial conditions and the Gaussian initial condition $\left|\Delta \Sigma\left(R ; f_{\mathrm{NL}}\right) / \Delta \Sigma\left(R ; f_{\mathrm{NL}}=0\right)-1\right|$ calculated from the curves shown in Fig. 4 . The dot-dashed, dashed, and dotted lines show $f_{\mathrm{NL}}=$ \pm 10 , \pm 50 , and \pm 100 , respectively, while the thin solid line shows $\propto R^{2}$ with an arbitrary normalization. 
noise of lens halos, as well as the shape noise $\sigma_{\gamma}$. As far as we know, this formula has not been derived before. Note that we have assumed a single source and lens redshift. For multiple source and lens redshifts, the covariance matrix needs to be suitably generalized.

Here, $C_{l}^{h}$ and $C_{l}^{\kappa}$ are the angular power spectra of the lens halos (galaxies or cluster of galaxies) and $\kappa$, respectively, and $n_{L}$ and $n_{S}$ are the number densities of the lens halos and the lensed (source) galaxies, respectively. These angular power spectra $C_{l}^{h \kappa}, C_{l}^{h}, C_{l}^{\kappa}$ will be related to the corresponding three-dimensional power spectrum, $P(k)$, in Sec III C.

In the limit that the cosmic variance is unimportant, we recover the usual expression used in the literature:

$$
\left\langle\bar{\gamma}_{t}^{h}(\theta) \bar{\gamma}_{t}^{h}\left(\theta^{\prime}\right)\right\rangle-\left\langle\bar{\gamma}_{t}^{h}(\theta)\right\rangle\left\langle\bar{\gamma}_{t}^{h}\left(\theta^{\prime}\right)\right\rangle=\frac{\sigma_{\gamma}^{2}}{N_{L}} \frac{\delta_{D}\left(\theta-\theta^{\prime}\right)}{2 \pi \theta n_{S}},
$$

where $N_{L}=4 \pi f_{\text {sky }} n_{L}$ is the total number of lens halos available in the data. In this limit the errors in different radial bins are uncorrelated, and they are simply given by the shape noise $\sigma_{\gamma}$ reduced by the square-root of the number of source galaxies available within each radial bin and the total number of lens halos that we can use for averaging the mean tangential shear. In particular, at each bin with a width $\Delta \theta$, we find the variance of

$$
\operatorname{Var}\left[\bar{\gamma}_{t}^{h}(\theta)\right]=\frac{\sigma_{\gamma}^{2}}{2 \pi \theta(\Delta \theta) n_{S} N_{L}},
$$

in the absence of the cosmic variance.

When would the cosmic variance become important? There is the maximum surface number density of sources $n_{S, \max }=\sigma_{\gamma}^{2} / C_{l}^{\kappa}$ above which the shape noise becomes irrelevant. This gives the maximum number of sources within a given radial bin of a width $\Delta \theta(\ll \theta)$ above which the shape noise becomes irrelevant:

$$
N_{S, \max }=2 \pi \theta(\Delta \theta) n_{S, \max }=(l \theta)^{2}\left(\frac{\Delta \theta}{\theta}\right) \frac{\sigma_{\gamma}^{2}}{l^{2} C_{l}^{\kappa} /(2 \pi)} .
$$

For $l \theta=\pi$ (the usual relation between $l$ and $\theta$ ) and $\sigma_{\gamma} \simeq$ 0.3 (realistic shape noise), we find

$$
N_{S, \max } \simeq\left(\frac{\Delta \theta}{\theta}\right) \frac{1}{l^{2} C_{l}^{\kappa} /(2 \pi)} .
$$

At $l \sim 100, l^{2} C_{l}^{\kappa} /(2 \pi) \sim 10^{-5}$ [50]; thus, we do not gain sensitivity any further by having more than, say, $10^{4}$ galaxies (for $\Delta \theta / \theta=0.1$ ) within a single radial bin.

Alternatively, one can define the minimum multipole $l_{\min }$ below which the cosmic variance term dominates:

$$
l_{\text {min }}=\sqrt{\frac{2 \pi n_{S}}{\sigma_{\gamma}^{2}} \frac{l^{2} C_{l}^{\kappa}}{2 \pi}} .
$$

For LSST, we expect to have the surface density of sources on the order of $n_{S}=30 \mathrm{arcmin}^{-2}=3.5 \times 10^{8} \mathrm{sr}^{-1}$. For $\sigma_{\gamma}=0.3$, we find $l_{\min }(\mathrm{LSST}) \sim 1.6 \times 10^{5} \sqrt{l^{2} C_{l}^{\kappa} /(2 \pi)}$. At $l \lesssim 10^{3}, l^{2} C_{l}^{\kappa} /(2 \pi) \lesssim 10^{-4}[50]$; thus, at $l \lesssim 10^{3}$ the cosmic variance term dominates.

In the limit that the covariance matrix is dominated by the cosmic variance terms, we have

$$
\begin{aligned}
& \left\langle\bar{\gamma}_{t}^{h}(\theta) \bar{\gamma}_{t}^{h}\left(\theta^{\prime}\right)\right\rangle-\left\langle\bar{\gamma}_{t}^{h}(\theta)\right\rangle\left\langle\bar{\gamma}_{t}^{h}\left(\theta^{\prime}\right)\right\rangle \\
& \quad=\frac{1}{4 \pi f_{\text {sky }}} \int \frac{l d l}{2 \pi} J_{2}(l \theta) J_{2}\left(l \theta^{\prime}\right) C_{l}^{h} C_{l}^{\kappa}\left(1+r_{l}^{2}\right),
\end{aligned}
$$

where $r_{l} \equiv C_{l}^{h \kappa} / \sqrt{C_{l}^{\kappa} C_{l}^{h}}$ is the cross-correlation coefficient. The variance at a given radial bin is

$$
\operatorname{Var}\left[\bar{\gamma}_{t}^{h}(\theta)\right]=\frac{1}{4 \pi f_{\text {sky }}} \int \frac{l d l}{2 \pi}\left[J_{2}(l \theta)\right]^{2} C_{l}^{h} C_{l}^{\kappa}\left(1+r_{l}^{2}\right) .
$$

\section{Detectability of the mean tangential shear}

In this section, we shall calculate the expected uncertainties in radially binned measurements of the mean tangential shear.

The mean tangential shear averaged within the $i$-th bin $\left\langle\hat{\bar{\gamma}}_{t}^{h}\right\rangle\left(\theta_{i}\right)$, i.e., the mean tangential shear averaged within an annulus between $\theta_{i, \min }$ and $\theta_{i, \max }$, is given by

$$
\left\langle\hat{\bar{\gamma}}_{t}^{h}\right\rangle\left(\theta_{i}\right)=\frac{2 \pi}{A\left(\theta_{i}\right)} \int_{\theta_{i, \text { min }}}^{\theta_{i, \text { max }}} \theta d \theta\left\langle\bar{\gamma}_{t}^{h}\right\rangle(\theta) \equiv \int \frac{l d l}{2 \pi} C_{l}^{h \kappa} \hat{J}_{2}\left(l \theta_{i}\right),
$$

where $A\left(\theta_{i}\right)=\pi\left(\theta_{i, \text { max }}^{2}-\theta_{i \text {, min }}^{2}\right)$ is the area of the annulus, and

$$
\hat{J}_{2}\left(l \theta_{i}\right)=\frac{2 \pi}{A\left(\theta_{i}\right)} \int_{\theta_{i, \text { min }}}^{\theta_{i, \max }} \theta d \theta J_{2}(l \theta)
$$

is the Bessel function averaged within a bin.

Similarly, the covariance matrix of the binned mean tangential shears is given by

$$
\begin{aligned}
C_{i j} \equiv & \left\langle\hat{\bar{\gamma}}_{t}^{h}\left(\theta_{i}\right) \hat{\bar{\gamma}}_{t}^{h}\left(\theta_{j}\right)\right\rangle-\left\langle\hat{\bar{\gamma}}_{t}^{h}\left(\theta_{i}\right)\right\rangle\left\langle\hat{\bar{\gamma}}_{t}^{h}\left(\theta_{j}\right)\right\rangle \\
= & \frac{1}{4 \pi f_{\text {sky }}} \int \frac{l d l}{2 \pi} \hat{J}_{2}\left(l \theta_{i}\right) \hat{J}_{2}\left(l \theta_{j}\right) \\
& \times\left[\left(C_{l}^{h \kappa}\right)^{2}+\left(C_{l}^{h}+\frac{1}{n_{L}}\right)\left(C_{l}^{\kappa}+\frac{\sigma_{\gamma}^{2}}{n_{S}}\right)\right] .
\end{aligned}
$$

This matrix contains the full information regarding the statistical errors of the binned measurements of the mean tangential shear, which includes the cosmic variance errors due to the cosmic shear $\left(C_{l}^{\kappa}\right)$, clustering of lens galaxies $\left(C_{l}^{h}\right)$ and their correlations $\left(C_{l}^{h \kappa}\right)$, the finite number density of lenses, and the noise in intrinsic shapes of source galaxies. 
The variance at a given radial bin is

$$
\begin{aligned}
\operatorname{Var}\left[\hat{\bar{\gamma}}_{t}^{h}\left(\theta_{i}\right)\right]= & \frac{1}{4 \pi f_{\text {sky }}} \int \frac{l d l}{2 \pi}\left[\hat{J}_{2}\left(l \theta_{i}\right)\right]^{2} \\
& \times\left[\left(C_{l}^{h \kappa}\right)^{2}+\left(C_{l}^{h}+\frac{1}{n_{L}}\right)\left(C_{l}^{\kappa}+\frac{\sigma_{\gamma}^{2}}{n_{S}}\right)\right] .
\end{aligned}
$$

In the analysis of the galaxy-galaxy-lensing effects in the literature, the cosmic variance due to cosmic shear is usually ignored:

$$
\begin{aligned}
\left.\operatorname{Var}\left[\hat{\bar{\gamma}}_{t}^{h}\left(\theta_{i}\right)\right]\right|_{\kappa=0}= & \frac{1}{4 \pi f_{\text {sky }}} \int \frac{l d l}{2 \pi}\left[\hat{J}_{2}\left(l \theta_{i}\right)\right]^{2} \\
& \times\left[\left(C_{l}^{h}+\frac{1}{n_{L}}\right) \frac{\sigma_{\gamma}^{2}}{n_{S}}\right] .
\end{aligned}
$$

This is probably a reasonable approximation for the current measurements at $R \lesssim 30 h^{-1} \mathrm{Mpc}$; however, on larger scales which will be probed by the next-generation lens surveys, the cosmic variance due to cosmic shear must be included, as we show in Fig. 6.

For estimating the expected uncertainties, we assume a million lens galaxies with very narrow (delta-functionlike) redshift distribution centered at $z_{L}\left(N_{L}=10^{6}\right)$ over the full sky $f_{\text {sky }}=1$. We also assume $\sigma_{\gamma}=0.3$, and $n_{S}=3.5 \times$ $10^{8} \mathrm{sr}^{-1}$. As the covariance matrix is dominated by the cosmic variance terms, the size of open boxes is insensitive to the exact values of $N_{L}, \sigma_{\gamma}$, or $n_{S}$. (See Sec. III C.) First, we calculate the binned uncertainties in the region close to the baryonic feature, $R \sim 110 h^{-1} \mathrm{Mpc}$. In Fig. 6, the open boxes show the full uncertainties including the cosmic variance due to cosmic shear [Eq. (28)], while the filled boxes show the uncertainties without the cosmic shear term [Eq. (29)]. The latter is clearly negligible compared to the former on large scales $R \gtrsim 50 h^{-1} \mathrm{Mpc}$.

Can we distinguish $\Delta \Sigma(R)$ with and without the baryonic feature? Without baryons, we do not see any features in $\Delta \Sigma(R)$; see dashed lines in Fig. 6 which are calculated from the smooth linear power spectrum without the baryonic feature [56]. To see if we can detect this feature in $\Delta \Sigma(R)$, we estimate the $\chi^{2}$ difference between $\Delta \Sigma(R)$ with and without the baryonic feature:

$$
\Delta \chi^{2} \equiv \sum_{i, j}\left(\Delta \Sigma_{i}-\Delta \Sigma_{i, \mathrm{nw}}\right) C_{i j}^{-1}\left(\Delta \Sigma_{j}-\Delta \Sigma_{j, \mathrm{nw}}\right),
$$

where $\Delta \Sigma_{i}$ is the mean tangential shear of $i$-th bin, $\Delta \Sigma_{\text {nw }}$ is $\Delta \Sigma$ without the baryonic feature, and $C_{i j}^{-1}$ is the inverse of the binned covariance matrix [Eq. (27)]. Note that we have to use the full covariance matrix as neighboring bins are strongly correlated (Fig. 7). Using only a single lens redshift slice, we find $\Delta \chi^{2}=0.85\left(z_{L}=0.3, b=2\right), 1.07$ $\left(z_{L}=0.5, b=2\right), 1.32\left(z_{L}=0.8, b=3\right)$, and $1.34\left(z_{L}=\right.$ $0.8, b=5)$. For example, if we add up all these measurements at different slices $\left(z_{L}=0.3,0.5\right.$, and 0.8$)$, significance of detection of the baryonic feature is $\Delta \chi^{2}=3.2$, i.e., 93\% C.L. As we expect to have many more lens

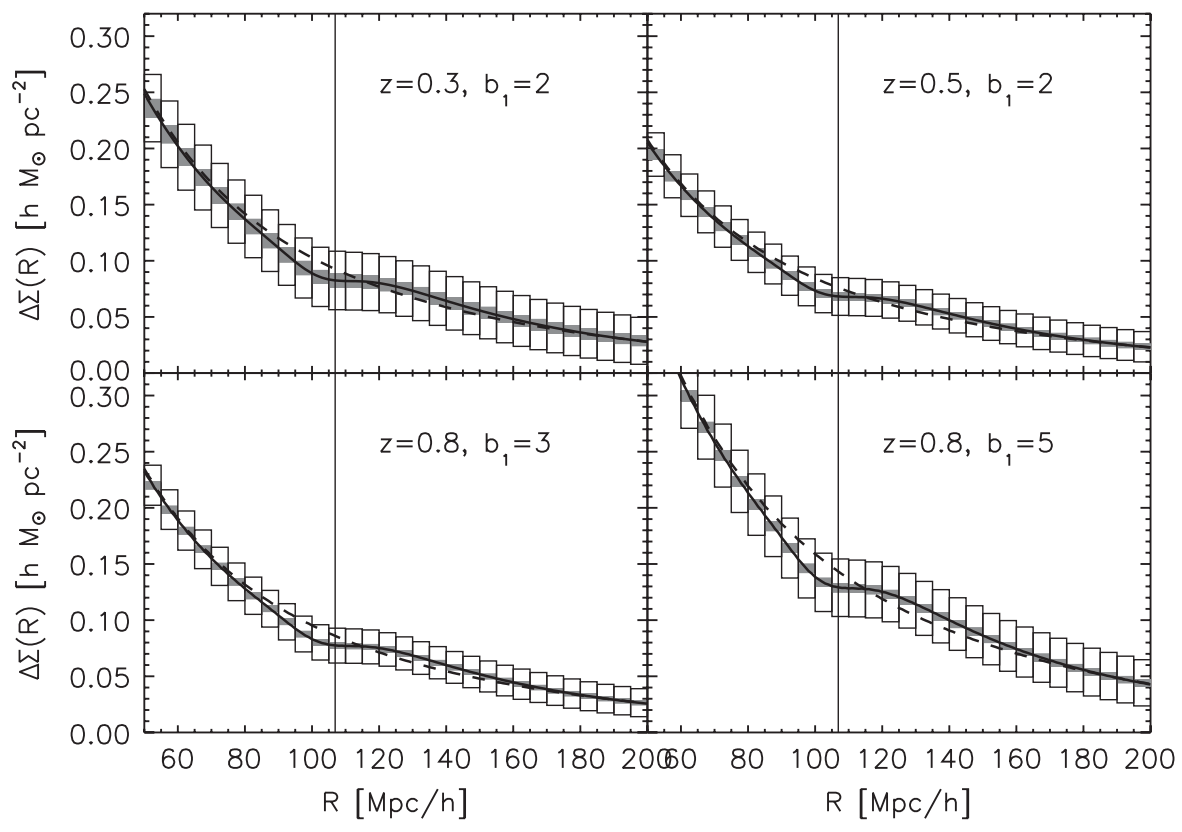

FIG. 6. Same as Fig. 3, but with the expected 1- $\sigma$ uncertainties for full-sky lens surveys and a single lens redshift. Adjacent bins are highly correlated, with the correlation coefficients shown in Fig. 7. The open (filled) boxes show the binned uncertainties with (without) the cosmic variance term due to the cosmic shear field included. See Eqs. (28) and (29) for the formulae giving open and filled boxes, respectively. We use the radial bin of size $\Delta R=5 h^{-1} \mathrm{Mpc}$. For comparison, we also show $\Delta \Sigma(R)$ computed from the smooth power spectrum without the baryonic feature [56] (dashed lines). Note that the uncertainties are calculated for a single lens redshift slice, and thus they will go down as we add more lens redshift slices. 

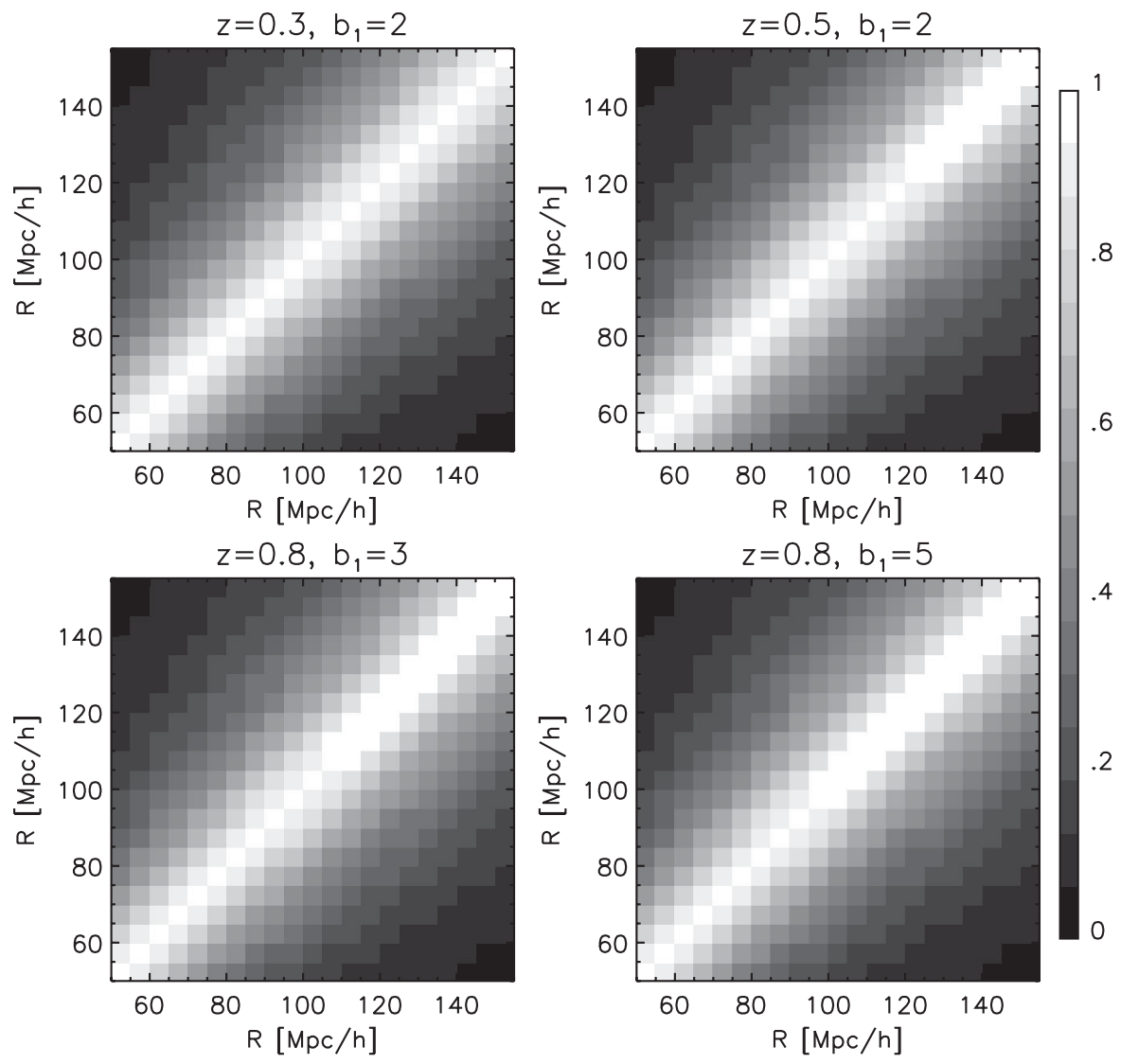

FIG. 7. The cross-correlation-coefficient matrix $r_{i j} \equiv C_{i j} / \sqrt{C_{i i} C_{j j}}$, where $C_{i j}$ is the covariance matrix given in Eq. (27), for a radial bin of $\Delta R=5 h^{-1} \mathrm{Mpc}$. We show $r_{i j}$ for the same populations of lens galaxies as shown in Figs. 3 and 6 . We use the same number of source galaxies and the same shape noise as in Fig. 6. The neighboring bins are highly correlated for $\Delta R<10 h^{-1} \mathrm{Mpc}$.

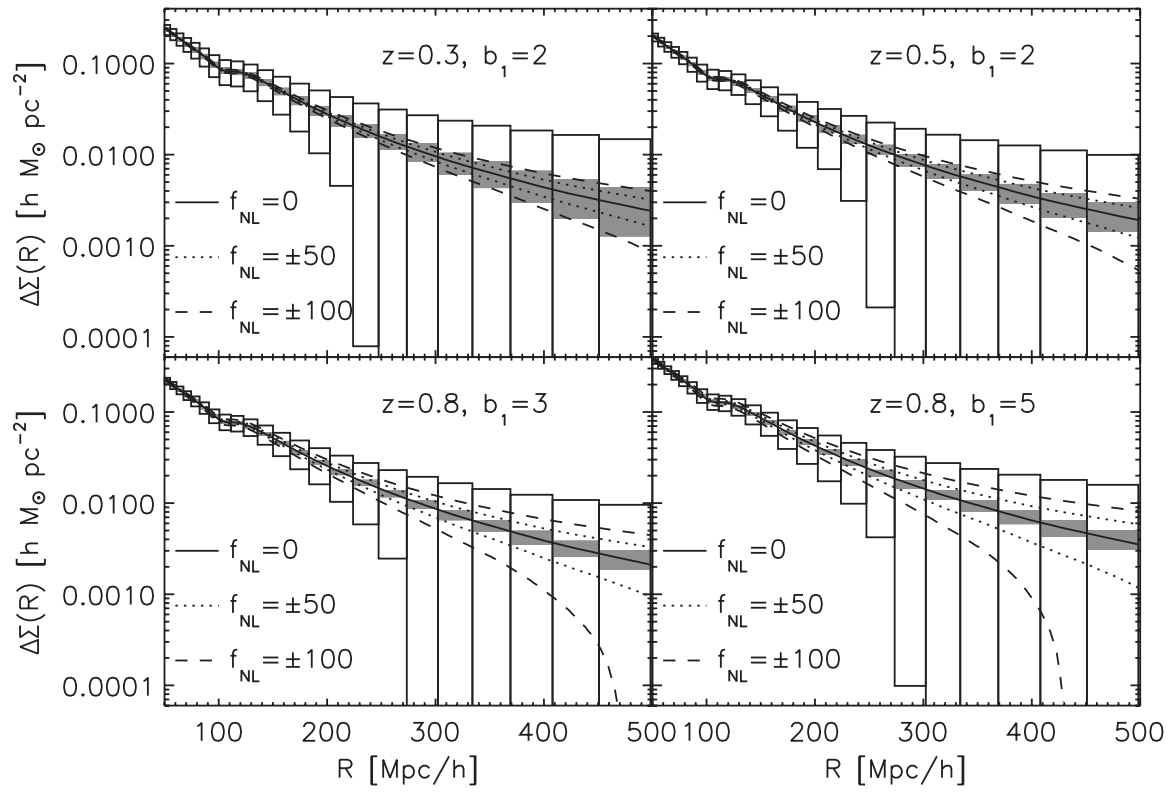

FIG. 8. Same as Fig. 4, but with the expected 1- $\sigma$ uncertainties for full-sky lens surveys and a single lens redshift. Adjacent bins are highly correlated. The open (filled) boxes show the binned uncertainties with (without) the cosmic variance term due to the cosmic shear field included. See Eqs. (28) and (29) for the formulae giving open and filled boxes, respectively. We use logarithmic bins with $\Delta R=R / 10$. Note that the uncertainties are calculated for a single lens redshift slice, and thus they will go down as we add more lens redshift slices. 
redshift slices from the future lens surveys, detection and measurement of the baryonic feature in $\Delta \Sigma$ are quite feasible. For multiple lens slices, the gain in the signalto-noise ratio will be approximately $\sqrt{N_{\text {lens }}}$; thus, for 10 lens slices the errors would be a factor of 3 smaller. At best, we can expect $\sim 25$ slices, which gives a factor of 5 reduction in errors.

What about $f_{\mathrm{NL}}$ ? We show the expected 1- $\sigma$ uncertainties for the mean tangential shears, $\Delta \Sigma(R)$, on larger scales in Fig. 8. For this figure, we use logarithmic bins with the radial size of $\Delta R / R=0.1$. We find that $\Delta \Sigma(R)$ on $R \simeq$ $250 h^{-1} \mathrm{Mpc}$ is detectable, even from a single lens redshift slice. This is remarkable; however, the predicted uncertainties are too large for us to distinguish between $f_{\mathrm{NL}}=0$ and $f_{\mathrm{NL}}=100$ using a single lens redshift slice. In order to obtain a tight limit on $f_{\mathrm{NL}}$, we would need to include many lens redshift slices.

Note that the uncertainty at a given $R$ is larger for a smaller lens redshift. This is because a given $R$ corresponds to a larger angular size for a lower lens redshift, making the cosmic variance contribution greater.

\section{HARMONIC SPACE APPROACH}

\section{A. Formula}

The mean tangential shear $\left\langle\bar{\gamma}_{t}^{h}\right\rangle$ or $\Delta \Sigma$ is currently widely used for measuring the halo-shear cross correlation, as this method is easy to implement and is less sensitive to systematic errors.

In this section, we shall study the effects of $f_{\mathrm{NL}}$ on the equivalent quantity in harmonic space: the haloconvergence cross-power spectrum, $C_{l}^{h \kappa}$. The mean tangential shear is related to $C_{l}^{h \kappa}$ by the two-dimensional Fourier integral given in Eq. (11).

The convergence field $\kappa(\mathbf{n})$ is the matter density fluctuations projected on the sky:

$$
\kappa(\mathbf{n})=\int_{0}^{\infty} d z W_{\kappa}(z) \delta_{m}\left[d_{A}(0 ; z) \mathbf{n}, z\right]
$$

where $\delta_{m}(\mathbf{r}, z) \equiv \rho_{m}(\mathbf{r}, z) / \bar{\rho}_{m}(z)-1$, and $W_{\kappa}(z)$ is a lens kernel which describes the efficiency of lensing for a given redshift distribution of sources $p\left(z_{S}\right)$ :

$$
W_{\kappa}(z)=\frac{\rho_{0}}{\Sigma_{c}\left(z ; z_{S}\right) H(z)},
$$

where the critical density $\Sigma_{c}$ is defined in Eq. (16).

Again using Limber's approximation (whose validity and limitation are studied in Appendix C), we find the relation between the angular cross-correlation power spectrum of the convergence field and the halo density at a given lens redshift $z_{L}, C_{l}^{h \kappa}\left(z_{L}\right)$, and the halo-mass crosscorrelation power spectrum at the same redshift $P_{h m}\left(k, z_{L}\right)$ as
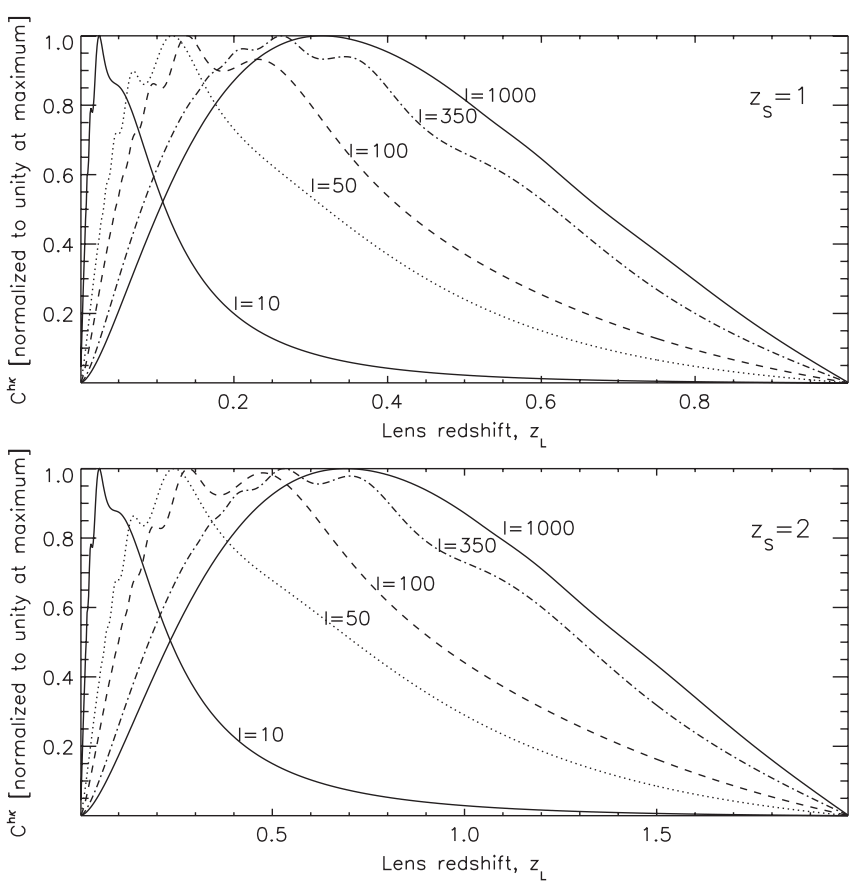

FIG. 9. Angular power spectrum of the galaxy-convergence cross correlation $C_{l}^{h \kappa}$ at various multipoles as a function of the lens redshift $z_{L}$ for two effective source redshifts $z_{s}=1$ (top) and 2 (bottom). We have divided $C_{l}^{h \kappa}$ by its maximum value. The solid, dotted, dashed, dot-dashed, and triple-dot-dashed lines show $l=10,50,100,350$, and 1000, respectively.

$$
\begin{aligned}
C_{l}^{h \kappa}\left(z_{L}\right)= & \frac{\rho_{0}}{\Sigma_{c}\left(z_{L} ; z_{S}\right) d_{A}^{2}\left(0 ; z_{L}\right)} P_{h m}\left[k=\frac{l+1 / 2}{d_{A}\left(0 ; z_{L}\right)}, z_{L}\right] \\
= & \frac{4 \pi G \rho_{0}}{c^{2}}\left(1+z_{L}\right) \frac{d_{A}\left(z_{L} ; z_{S}\right)}{d_{A}\left(0 ; z_{L}\right) d_{A}\left(0 ; z_{S}\right)} \\
& \times P_{h m}\left[k=\frac{l+1 / 2}{d_{A}\left(0 ; z_{L}\right)}, z_{L}\right]
\end{aligned}
$$

Figure 9 shows $C_{l}^{h \kappa}\left(z_{L}\right)$ for the Gaussian density field as a function of lens redshifts $z_{L}$. The convergence fields at low (high) multipoles are better correlated with low- $z$ (high- $z$ ) galaxies. This is due to the shape of the matter power spectrum: on very large scales (i.e., low $l$ ), the matter power spectrum is given by the initial power spectrum $P_{h m}(k) \propto k$, and thus we get $1 / d_{A}\left(0 ; z_{L}\right)$ from $P_{h m}\left[k=l / d_{A}\left(0 ; z_{L}\right)\right]$. This gives a larger weight to low- $z$ galaxies. On smaller scales where $P_{h m}(k) \propto k^{n_{\text {eff }}}$ with $n_{\text {eff }} \simeq-3$, we get positive powers of $d_{A}\left(0 ; z_{L}\right)$ from $P_{h m}\left[k=l / d_{A}\left(0 ; z_{L}\right)\right]$, which gives a larger weight to high- $z$ galaxies.

\section{B. Result}

We can now calculate $C_{l}^{h \kappa}$ for various values of $f_{\mathrm{NL}}$. We use 


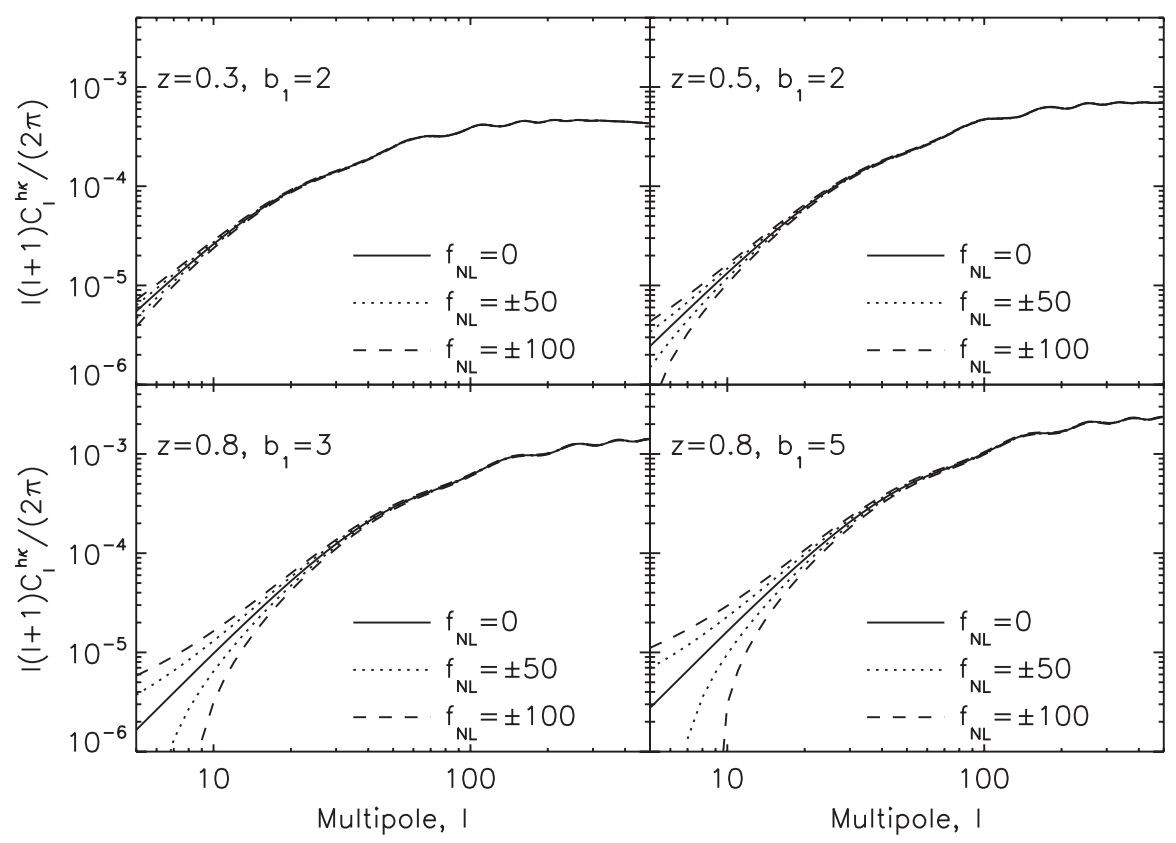

FIG. 10. Imprints of the local-type primordial non-Gaussianity in the galaxy-convergence cross-power spectrum, $l(l+1) C_{l}^{h \kappa} /(2 \pi)$, for the same populations of lens galaxies as in Fig. 3 . The solid, dashed, and dotted lines show $f_{\mathrm{NL}}=0$, \pm 50 , and \pm 100 , respectively.

$$
\begin{aligned}
C_{l}^{h \kappa}\left(z_{L}\right)= & \frac{4 \pi G \rho_{0}}{c^{2}}\left(1+z_{L}\right) \frac{d_{A}\left(z_{L} ; z_{S}\right)}{d_{A}\left(0 ; z_{L}\right) d_{A}\left(0 ; z_{S}\right)} \\
& \times\left[b_{1}\left(z_{L}\right)+\Delta b\left(k=\frac{l+1 / 2}{d_{A}\left(0 ; z_{L}\right)}, z_{L}\right)\right] \\
& \times P_{m}\left[k=\frac{l+1 / 2}{d_{A}\left(0 ; z_{L}\right)}, z_{L}\right]
\end{aligned}
$$

where the scale-dependent bias $\Delta b(k, z)$ is given by Eq. (3).

Figure 10 shows $C_{l}^{h \kappa}\left(z_{L}\right)$ for $f_{\mathrm{NL}}= \pm 50$ and \pm 100 for populations of galaxies that we have considered in the previous sections. For each lens redshift, we calculate the "effective" source redshift by requiring that the angular diameter distance to the source redshift is twice as large as

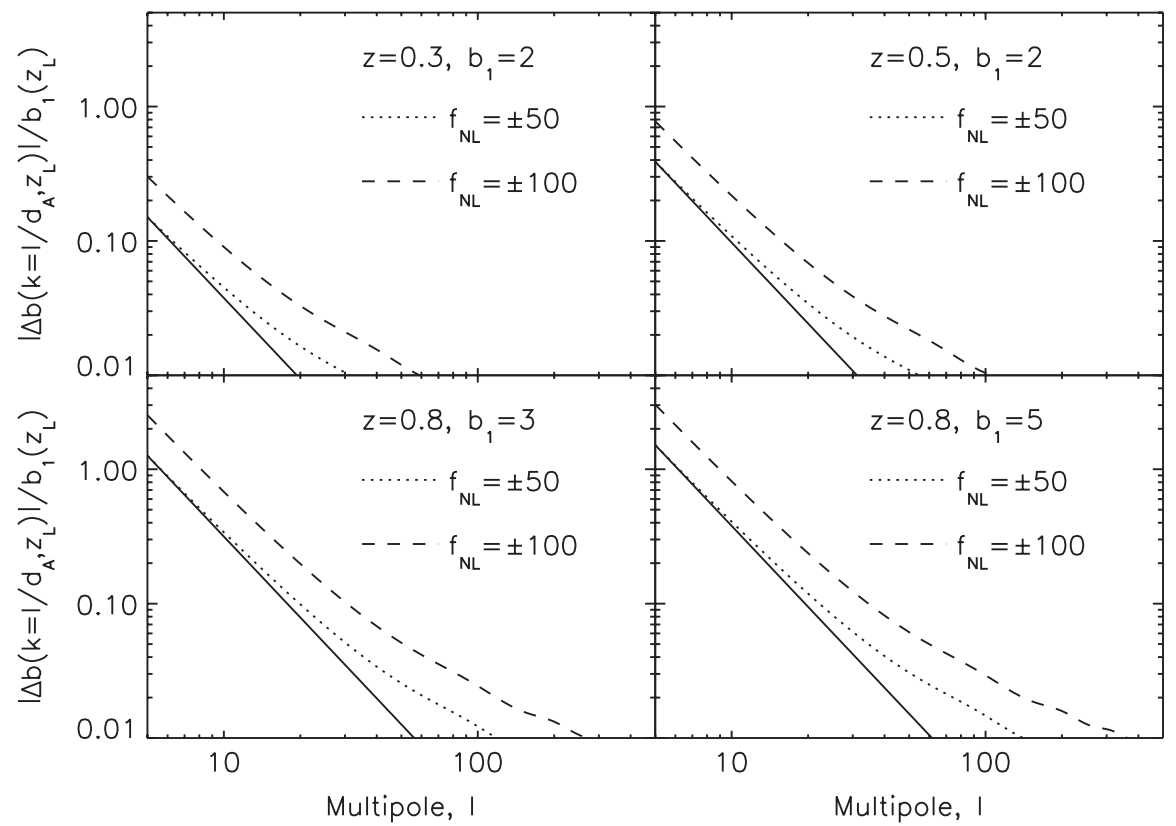

FIG. 11. Fractional differences between $C_{l}^{h \kappa}$ from non-Gaussian initial conditions and the Gaussian initial condition, calculated from the curves shown in Fig. 10. These differences are equal to $\left|\Delta b\left(l=k / d_{A}, z_{L}\right)\right| / b_{1}\left(z_{L}\right)$. The dashed and dotted lines show $f_{\mathrm{NL}}= \pm 50$ and \pm 100 , respectively, while the thin solid lines show $l^{-2}$ with an arbitrary normalization. 
that to the lens redshift, i.e., $d_{A}\left(0 ; z_{S}\right)=2 d_{A}\left(0 ; z_{L}\right)$. With this requirement, the source redshifts are $z_{s}=0.65,1.19$, and 2.25 for $z_{L}=0.3,0.5$, and 0.8 , respectively.

Figure 11 shows the fractional differences between nonGaussian predictions and the Gaussian prediction $\left(f_{\mathrm{NL}}=\right.$ $0)$, which are simply equal to $\Delta b\left(k, z_{L}\right) / b_{1}\left(z_{L}\right)$ where $k=$ $l / d_{A}\left(0 ; z_{L}\right)$. As expected from the form of the scaledependent bias, the difference grows toward small multipoles as roughly $1 / l^{2}$. While lower redshift populations do not show more than $10 \%$ difference at $l \geq 10$ for $f_{\mathrm{NL}}=$ \pm 50 , a higher- $z$ population of lens galaxies or clusters of galaxies at $z_{L}=0.8$ show the differences at the level of $\sim 10 \%$ at $l \sim 20$ and $\sim 30 \%$ at $l \sim 10$. Are these effects detectable?

\section{Covariance matrix of the galaxy-convergence cross-power spectrum}

The covariance matrix of the galaxy-convergence crosscorrelation power spectrum is given by

$$
\begin{aligned}
& \left\langle C_{l}^{h \kappa} C_{l^{\prime}}^{h \kappa}\right\rangle-\left\langle C_{l}^{h \kappa}\right\rangle\left\langle C_{l^{\prime}}^{h \kappa}\right\rangle \\
& =\frac{\delta_{l l^{\prime}}}{(2 l+1) f_{\text {sky }}}\left[\left(C_{l}^{h \kappa}\right)^{2}+\left(C_{l}^{h}+\frac{1}{n_{L}}\right)\left(C_{l}^{\kappa}+\frac{\sigma_{\gamma}^{2}}{n_{S}}\right)\right],
\end{aligned}
$$

where $\delta_{l l^{\prime}}$ is Kronecker's delta symbol showing that the angular power spectra at different multipoles are uncorrelated. Again, $C_{l}^{h}$ and $C_{l}^{\kappa}$ are the angular power spectra of the lens halos (galaxies or cluster of galaxies) and $\kappa$, respectively, and $n_{L}$ and $n_{S}$ are the number densities of the lens halos and the lensed (source) galaxies, respectively.

We calculate $C_{l}^{\kappa}$ by using Limber's approximation as

$$
C_{l}^{\kappa}=\int_{0}^{z_{S}} d z \frac{\rho_{0}^{2}}{\sum_{c}^{2}\left(z ; z_{S}\right)} \frac{P_{m}\left[k=\frac{l+1 / 2}{d_{A}(0 ; z)} ; z\right]}{H(z) d_{A}^{2}(0 ; z)} .
$$

However, we cannot use Limber's approximation for $C_{l}^{h}$ unless one considers lens redshift slices that are broad. As we are assuming a thin lens redshift slice throughout this paper, we must not use Limber's approximation, but evaluate the exact integral relation:

$$
C_{l}^{h}=\frac{2}{\pi} \int d k k^{2} P_{g}\left(k, z_{L}\right) j_{l}^{2}\left[k d_{A}\left(z_{L}\right)\right]
$$

where $j_{l}$ is the spherical Bessel function, and $P_{g}(k, z)$ is the linear galaxy power spectrum: $P_{g}(k)=b_{1}^{2} P_{m}(k)$.

Figure 12 shows the galaxy-galaxy, galaxy-convergence, and convergence-convergence angular power spectra for Gaussian $\left(f_{\mathrm{NL}}=0\right)$ initial conditions. We also show the shot noise of the galaxy angular power spectrum, $1 / n_{L}$, and the shape noise of the convergence power spectrum, $\sigma_{\gamma}^{2} / n_{S}$, with the following representative values: $N_{L}=$ $4 \pi n_{L}=10^{6}, n_{S}=3.5 \times 10^{8} \mathrm{sr}^{-1}$, and $\sigma_{\gamma}=0.3$. We find $1 / n_{L} \ll C_{l}^{h}$ and $\sigma_{\gamma}^{2} / n_{S} \ll C_{l}^{\kappa}$ for the multipoles that we are interested in, i.e., $l \lesssim 100$, and thus we conclude that the uncertainties are totally dominated by the cosmic

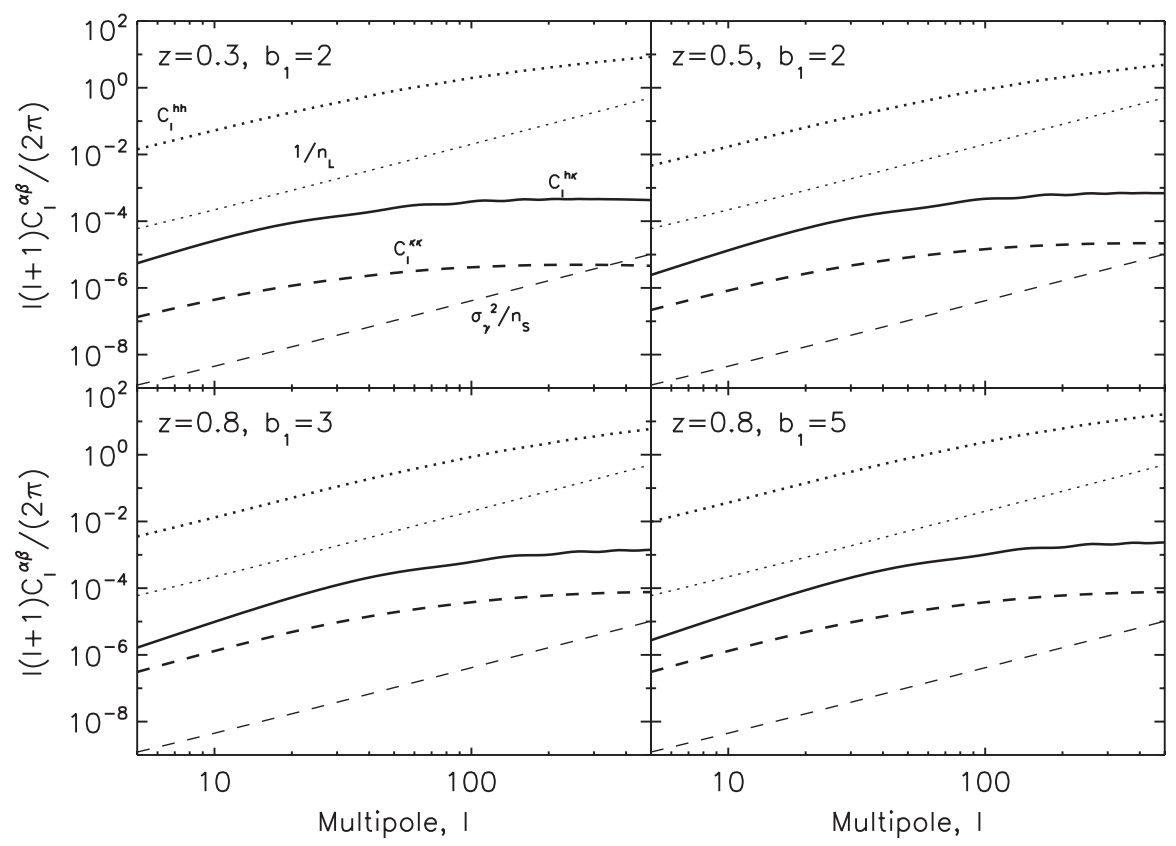

FIG. 12. Angular power spectra of the galaxy-galaxy correlation $C_{l}^{h}$ (thick dotted lines), the galaxy-convergence cross-correlation $C_{l}^{h \kappa}$ (thick solid lines), and the convergence-convergence correlation $C_{l}^{\kappa}$ (thick dashed lines) for the Gaussian initial condition $\left(f_{\mathrm{NL}}=\right.$ 0 ). The four panels show the same populations of galaxies and clusters of galaxies as in Fig. 10. We also show the galaxy shot noise $1 / n_{L}$ (thin dotted lines) as well as the source shape noise $\sigma_{\gamma}^{2} / n_{S}$ (thin dashed lines) for $N_{L}=10^{6}, \sigma_{\gamma}=0.3$, and $n_{S}=3.5 \times 10^{8} \mathrm{sr}^{-1}$. We find $1 / n_{L} \ll C_{l}^{h}$ and $\sigma_{\gamma}^{2} / n_{S} \ll C_{l}^{\kappa}$ for $l \lesssim 100$. 
variance terms. In other words, the size of the uncertainties are insensitive to the exact choices of $N_{L}, \sigma_{\gamma}$, or $n_{S}$.

We also find that the values of cross-correlation coefficients $r_{l} \equiv C_{l}^{h \kappa} / \sqrt{C_{l}^{h} C_{l}^{\kappa}}$ are small (of order 10-20\%): the maximum values are $0.19,0.15$, and 0.13 for $z_{L}=0.3,0.5$, and 0.8 , respectively. This implies that one may ignore the contribution of $C_{l}^{h \kappa}$ to the covariance matrix, approximating the variance of $C_{l}^{h \kappa}$ of a single lens redshift slice for a multipole bin of size $\Delta l$ as:

$$
\operatorname{Var}\left(C_{l}^{h \kappa}\right)=\frac{C_{l}^{h} C_{l}^{\kappa}}{(2 l+1) \Delta l f_{\mathrm{sky}}}
$$

Therefore, we should be able to measure the galaxyconvergence cross-power spectrum with $C_{l}^{h \kappa} / \sqrt{\operatorname{Var}\left(C_{l}^{h \kappa}\right)} \gtrsim 1$ when the multipoles satisfy

$$
l \gtrsim l_{\min } \equiv \frac{1}{r_{l} \sqrt{2(\Delta l / l) f_{\text {sky }}}} .
$$

For the galaxy-convergence power spectra in Fig. 10 with the full-sky coverage $\left(f_{\text {sky }}=1\right)$ and $\Delta l / l=0.23$, we find $l_{\text {min }}=9.0,12.1$, and 15.7 for $z_{L}=0.3\left(z_{S}=0.65\right), 0.5$ (1.19), and $0.8(2.25)$, respectively.

Similarly, we can estimate the maximum radius below which we can measure the mean tangential shear $\Delta \Sigma(R)$ as

$$
R_{\max } \simeq \frac{\pi d_{A}\left(0 ; z_{L}\right)}{l_{\min }}
$$

For example, with $\Delta R / R=\Delta l / l=0.1$, we get $R_{\max } \simeq$ 215, 260, and $300 h^{-1} \mathrm{Mpc}$ for $z_{L}=0.3,0.5$, and 0.8 , respectively. These values do give the radii at which the signal-to-noise ratios are roughly unity in Fig. 8.

Figure 13 shows the expected $1-\sigma$ uncertainties of $C_{l}^{h \kappa}$ for several populations of lens galaxies. We find that the cosmic variance completely dominates the uncertainties on large scales (low $l$ ) where the non-Gaussian effects are the largest. Again, while we find that it would be difficult to measure $f_{\mathrm{NL}}$ from a single lens redshift slice, combining many redshift slices should help us measure $f_{\mathrm{NL}}$, especially when we can use many slices at moderately high redshifts.

\section{HALO-MASS CORRELATION FROM GALAXY-CMB LENSING}

\section{A. Formula}

Instead of using the background galaxies for measuring the cosmic shear field due to the intervening mass, one can use the $\mathrm{CMB}$ as the background light and measure the shear field of the $C M B$ lensing due to the intervening mass between us and the photon decoupling epoch at $z_{*} \simeq$ 1089. See [57] for a review on the CMB lensing.

The lensing effect makes CMB anisotropies (both temperature and polarization) non-Gaussian by producing a

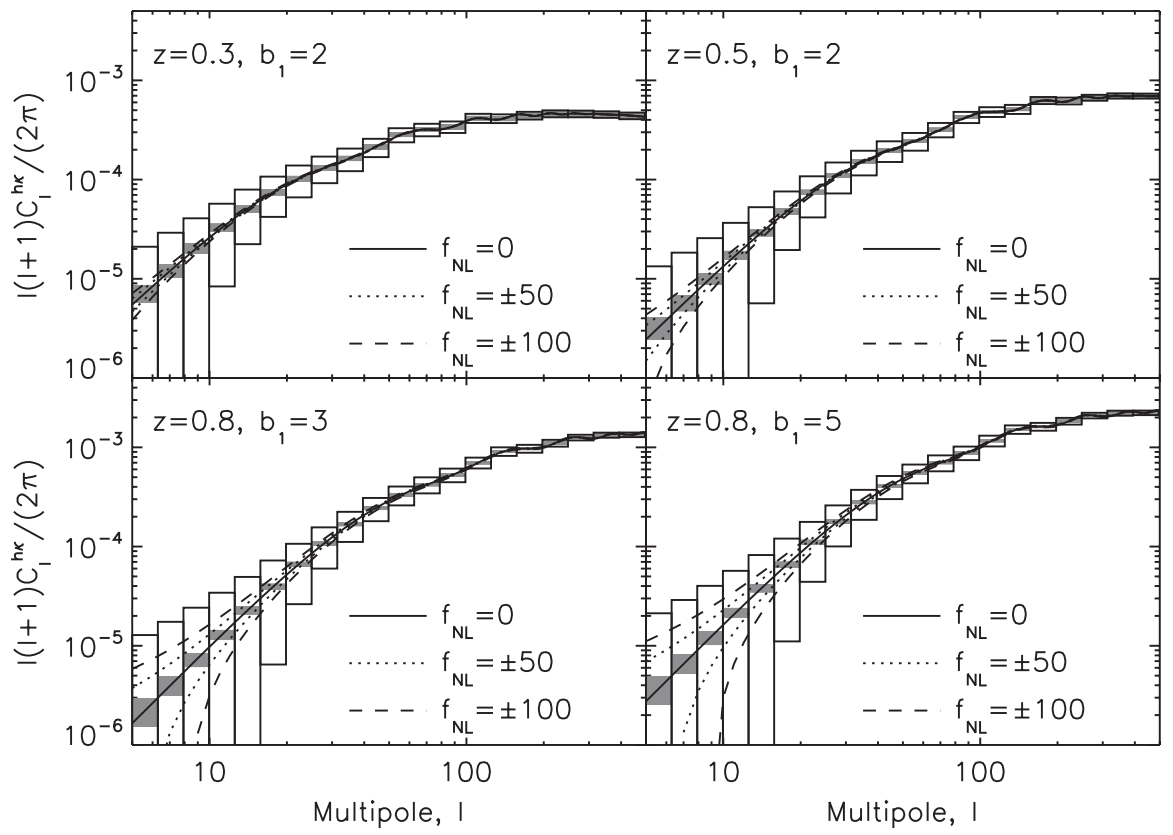

FIG. 13. Same as Fig. 10, with the expected 1- $\sigma$ uncertainties for full-sky lens surveys and a single lens redshift. Adjacent bins are uncorrelated. The open (filled) boxes show the binned uncertainties with (without) the cosmic variance term due to the cosmic shear field included. We used Eq. (34) for the open boxes, and Eq. (34) with $C_{l}^{h \kappa}=0=C_{l}^{\kappa}$ for the filled boxes. We use logarithmic bins of $\Delta l=0.23 l$. Note that the uncertainties are calculated for a single lens redshift slice, and thus they will go down as we add more lens redshift slices. 
nonvanishing connected four-point function, although it does not produce any nonvanishing three-point function. One can use this property to reconstruct the lensing potential field, hence the projected mass-density field between us and $z_{*}$, from the four-point function of CMB [58-60].

By cross correlating the halo over-density field $\delta_{h}$ at some redshift $z_{L}$ (measured from spectroscopic observations) and the $\kappa$ field reconstructed from the CMB lensing, one can measure the halo-convergence angular power spectrum $C_{l}^{h \kappa}$.

The angular power spectrum of the galaxy-CMB lensing cross correlation is merely a special case of the galaxyconvergence cross correlation that we have studied in the previous section: all we need to do is to set the source redshift $z_{S}$ to be the redshift of the photon decoupling epoch $z_{*} \simeq 1089$, i.e., $z_{S}=z_{*}$. Note that for a flat universe, $\quad d_{A}\left(z_{L} ; z_{*}\right)=d_{A}\left(0 ; z_{*}\right)-d_{A}\left(0 ; z_{L}\right) \quad$ where $d_{A}\left(0 ; z_{*}\right)=9.83 h^{-1} \mathrm{Gpc}$.

Figure 14 shows that the CMB lensing at low (high) multipoles are better correlated with low- $z$ (high- $z$ ) galaxies. This is due to the shape of the matter power spectrum, as we have explained in the previous section. Note that $C_{l}^{h \kappa}$ of the CMB lensing for a given multipole decreases more slowly with $z_{L}$ than that of the galaxy lensing due to the geometrical factor $d_{A}\left(z_{L} ; z_{S}\right) / d_{A}\left(0 ; z_{S}\right)$.

Note that CMB and galaxies at $z \lessgtr 1$ are correlated also via the integrated Sachs-Wolfe (ISW) effect [61]. We shall not include this effect in our cross-correlation calculation for the following reason. We calculate the cross-correlation signal between galaxies and the convergence field reconstructed from $\mathrm{CMB}$. This reconstruction relies on the fact that lensed CMB fluctuations have nonvanishing connected four-point function. On the other hand, the linear integrated

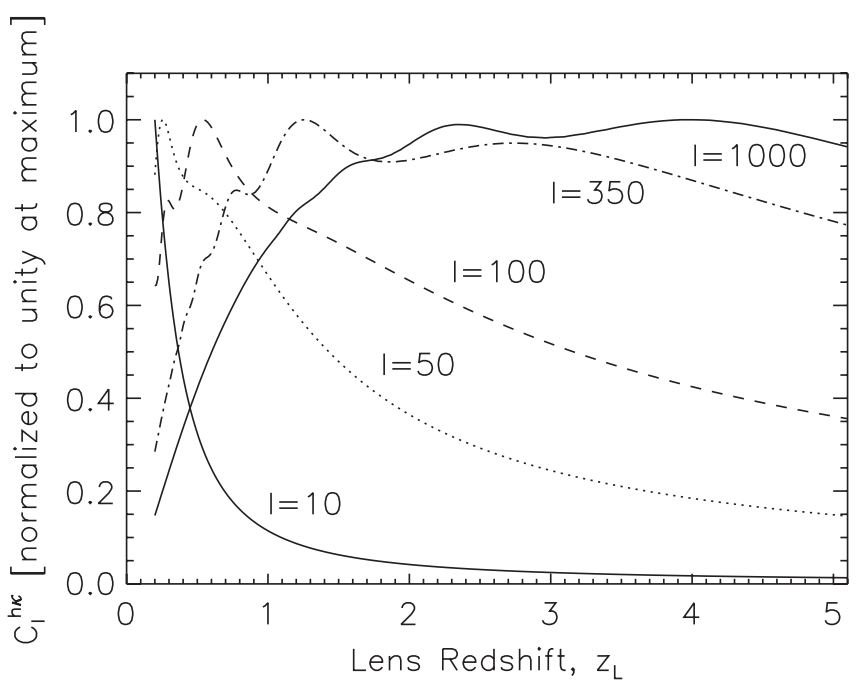

FIG. 14. Angular power spectrum of the galaxy-CMB lensing $C_{l}^{h \kappa}$ at various multipoles as a function of the lens redshift $z_{L}$. We have divided $C_{l}^{h \kappa}$ by its maximum value. The solid, dotted, dashed, dot-dashed, and triple-dot-dashed lines show $l=10,50$, 100,350 , and 1000, respectively.
Sachs-Wolfe effect does not have such a particular form of four-point function induced by lensing, and thus should not contribute to the reconstructed convergence field. See [34] for the effects of $f_{\mathrm{NL}}$ on the galaxy-integrated Sachs-Wolfe cross correlation.

\section{B. Results}

We can now calculate $C_{l}^{h \kappa}$ for various values of $f_{\mathrm{NL}}$. We use

$$
\begin{aligned}
C_{l}^{h \kappa}\left(z_{L}\right)= & \frac{4 \pi G \rho_{0}}{c^{2}}\left(1+z_{L}\right) \frac{d_{A}\left(z_{L} ; z_{*}\right)}{d_{A}\left(0 ; z_{L}\right) d_{A}\left(0 ; z_{*}\right)} \\
& \times\left[b_{1}\left(z_{L}\right)+\Delta b\left(k=\frac{l+\frac{1}{2}}{d_{A}\left(0 ; z_{L}\right)}, z_{L}\right)\right] \\
& \times P_{m}\left[k=\frac{l+\frac{1}{2}}{d_{A}\left(0 ; z_{L}\right)}, z_{L}\right]
\end{aligned}
$$

where the scale-dependent bias $\Delta b(k, z)$ is given by Eq. (3).

Figure 15 shows $C_{l}^{h \kappa}\left(z_{L}\right)$ for $f_{\mathrm{NL}}= \pm 50$ and \pm 100 for populations of low $-z$ galaxies that we have considered in the previous sections: $b_{1}=2$ at $z_{L}=0.3$ (similar to SDSS LRGs, top-left), $b_{1}=2$ at $z_{L}=0.5$ (higher- $z$ LRGs, topright), $b_{1}=2$ at $z_{L}=0.8$ (galaxies that can be observed by LSST, bottom-left), and $b_{1}=5$ at $z_{L}=0.8$ (clusters of galaxies that can be observed by LSST, bottom-right). The fractional differences between non-Gaussian predictions and the Gaussian prediction $\left(f_{\mathrm{NL}}=0\right)$ are exactly the same as those shown in Fig. 11: in the limit where Limber's approximation is valid, the galaxy-convergence power spectrum and the galaxy-CMB lensing power spectrum for the same lens galaxies differ only by a constant geometrical factor of $d_{A}\left(z_{L} ; z_{*}\right) d_{A}\left(0 ; z_{S}\right) /$ $d_{A}\left(z_{L} ; z_{S}\right) d_{A}\left(0 ; z_{*}\right)$. Incidentally, for our choice of the source redshifts in the previous section, $2 d_{A}\left(z_{L} ; z_{*}\right) / d_{A}\left(0 ; z_{*}\right)=1.83,1.73$, and 1.60 for $z_{L}=0.3$, 0.5 , and 0.8 , respectively.

Therefore, the galaxy-CMB lensing cross correlation would provide a nice cross-check for systematics of the galaxy-convergence cross correlation, and vice versa: after all, we are measuring the same quantity $P_{h m}(k)$ by two different background sources, high- $z$ galaxies and CMB.

In using high- $z$ galaxies as sources, the galaxy-galaxy lensing measurement may be susceptible to systematic errors widely discussed in the lensing literature, namely, shear calibration, coherent point spread function (PSF) anisotropy, redshift biases, magnification bias, and intrinsic alignments of galaxies. Here, we are particularly concerned with errors that affect galaxy-shear cross correlations by mimicking the angular dependence of the signal due to nonzero $f_{\mathrm{NL}}$. Fortunately, most systematic errors that affect shear-shear correlations do not contribute to galaxy-shear cross correlations: for instance, point spread function anisotropy affects background galaxy shapes but not foreground galaxy locations [62]. With 


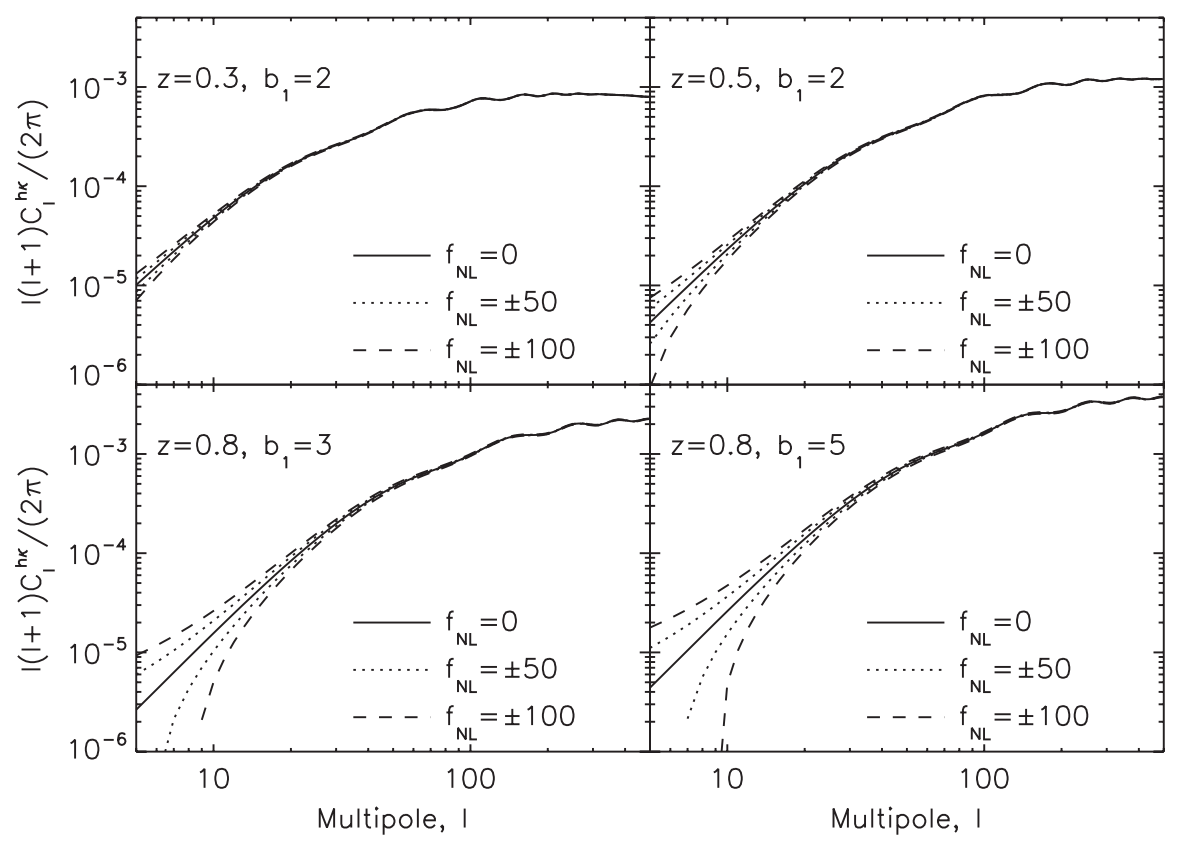

FIG. 15. Imprints of the local-type primordial non-Gaussianity in the galaxy-CMB lensing power spectrum $l(l+1) C_{l}^{h \kappa} /(2 \pi)$ for the same populations of lens galaxies as in Fig. 3 . The solid, dashed, and dotted lines show $f_{\mathrm{NL}}=0$, \pm 50 , and \pm 100 , respectively.

standard lensing data analysis methods, it can be ensured that both the shear calibration and point spread function do not contribute a scale dependence to the first order. Biases in the redshift distributions of lens and source galaxies can similarly lead to a misestimation of the amplitude of the signal, but not its scale dependence. Thus, to the lowest order, the measurement of $f_{\mathrm{NL}}$ via the scale dependence of the galaxy-galaxy lensing signal is robust to the leading systematic errors in weak lensing. But a detailed study of various sources of error is needed given the small signal we are seeking.

Another benefit of using the CMB lensing as a proxy for the intervening matter distribution is that we can probe the galaxy-matter cross correlation at high redshift to which we cannot reach with the galaxy-galaxy lensing method. It is especially useful for probing primordial non-

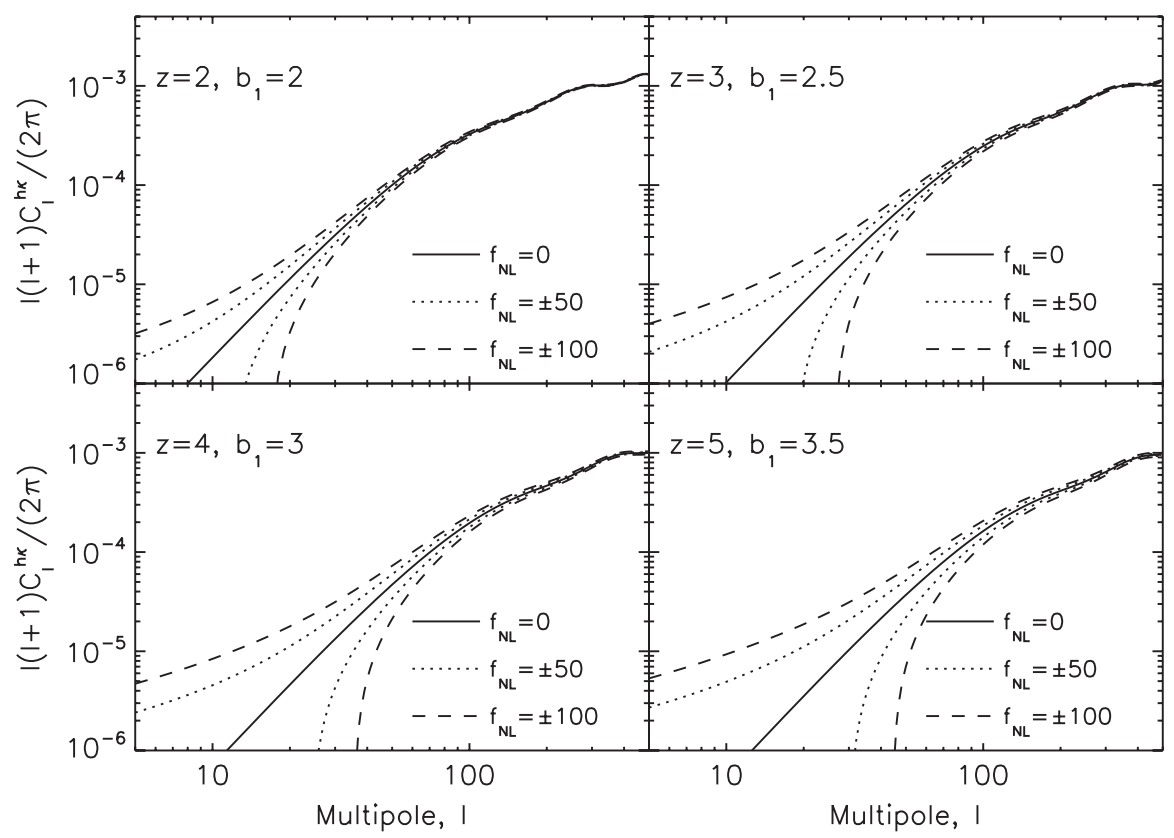

FIG. 16. Same as Fig. 15, but for high- $z$ lens galaxies with $b_{1}=2$ at $z_{L}=2$ (top-left), $b_{1}=2.5$ at $z_{L}=3$ (top-right), $b_{1}=3$ at $z_{L}=4$ (bottom-left), and $b_{1}=3.5$ at $z_{L}=5$ (bottom-right). 


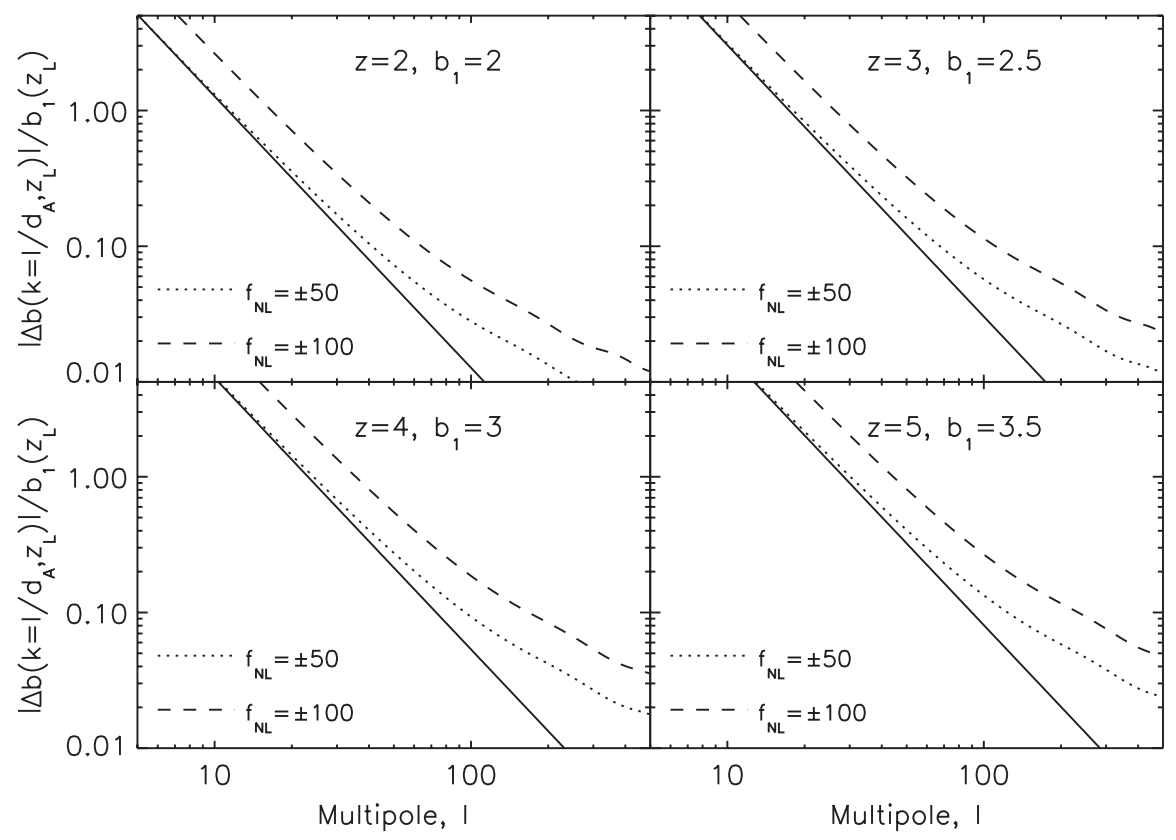

FIG. 17. Same as Fig. 11, but for high- $z$ lens galaxies with $b_{1}=2$ at $z_{L}=2$ (top-left), $b_{1}=2.5$ at $z_{L}=3$ (top-right), $b_{1}=3$ at $z_{L}=4$ (bottom-left), and $b_{1}=3.5$ at $z_{L}=5$ (bottom-right).

Gaussianity, as the scale-dependent bias signal is higher for higher lens redshift: $\Delta b\left(k, z_{L}\right) \propto 1 / D\left(z_{L}\right)$ [see Eq. (3)]. Therefore, we find that even higher- $z$ populations of galaxies give us a much better chance of detecting the effects of $f_{\mathrm{NL}}$. Figure 16 shows $C_{l}^{h \kappa}\left(z_{L}\right)$ for $f_{\mathrm{NL}}= \pm 50$ and \pm 100 for populations of high- $z$ galaxies: $b_{1}=2$ at $z_{L}=2$ (topleft), $b_{1}=2.5$ at $z_{L}=3$ (top-right), $b_{1}=3$ at $z_{L}=4$ (bottom-left), and $b_{1}=3.5$ at $z_{L}=5$ (bottom-right). The first one, a spectroscopic galaxy survey at $z_{L}=2$ with $b_{L}=2$, is within reach by, e.g., the Hobby-Eberly Telescope Dark Energy Experiment (HETDEX) [63,64]. There we find, for $f_{\mathrm{NL}}= \pm 50, \sim 10 \%$ effect at $l \sim 40$, and a factor of 2 effect at $l \sim 10$ (see Fig. 17). The effects grow bigger at higher $z$ : higher- $z$ surveys at $z>3$ can be done with, e.g., the concept of the Cosmic Inflation Probe (CIP). ${ }^{5}$ At $z_{L}=4$ and 5 (with $b_{1}=3$ and 4 , respectively) we find $\sim 10 \%$ effect at $l \sim 100$, a factor of 2 effect at $l \sim$ 30 , and even bigger effects at $l \lesssim 30$ (see Fig. 17).

\section{Covariance matrix of the galaxy-CMB lensing}

The covariance matrix of the galaxy-CMB lensing is given by [65]

$$
\begin{aligned}
& \left\langle C_{l}^{h \kappa} C_{l^{\prime}}^{h \kappa}\right\rangle-\left\langle C_{l}^{h \kappa}\right\rangle\left\langle C_{l^{\prime}}^{h \kappa}\right\rangle \\
& =\frac{\left(C_{l}^{h \kappa}\right)^{2}+\left(C_{l}^{h}+1 / n_{L}\right)\left(C_{l}^{\kappa}+N_{l}^{\kappa}\right)}{(2 l+1) f_{\text {sky }}} \delta_{l l^{\prime}},
\end{aligned}
$$

where $N_{l}^{\kappa}$ is the reconstruction noise from CMB given by [58]. The covariance matrix equation here is the same as Eq. (34), except that now the shape noise of source galaxies

\footnotetext{
${ }^{5}$ http://www.cfa.harvard.edu/cip/
}

is replaced by the reconstruction noise of CMB lensing. In what follows, we shall assume a "nearly perfect" CMB experiment considered in $\mathrm{Hu}$ and Okamoto [58], whose Gaussian random detector noise is modeled as [66]

$$
\begin{aligned}
& \left.C_{l}^{T}\right|_{\text {noise }}=\left(\frac{T_{\mathrm{CMB}}}{\Delta_{T}}\right)^{-2} e^{l(l+1) \sigma^{2} / 8 \ln 2,} \\
& \left.C_{l}^{E}\right|_{\text {noise }}=\left.C_{l}^{B}\right|_{\text {noise }}=\left(\frac{T_{\mathrm{CMB}}}{\Delta_{T}}\right)^{-2} e^{l(l+1) \sigma^{2} / 8 \ln 2,}
\end{aligned}
$$

where the white noise level of detectors is $\Delta_{T}=\Delta_{P} / \sqrt{2}=$ $1 \mu \mathrm{K}$ arcmin, and the full-width-at-half-maximum (FWHM) of the beam is $\sigma=4^{\prime}$. With these detector parameters and the cosmological parameters of the "WMAP + BAO + SN ML" parameters in Table 1 of [2], we find $N_{l}^{\kappa} \simeq 6 \times 10^{-8} \mathrm{sr}^{-1}$ on large scales, $l<100$.

Figure 18 shows the galaxy-galaxy, galaxy-convergence, convergence-convergence angular power spectra for the Gaussian initial condition $\left(f_{\mathrm{NL}}=0\right)$. This figure is qualitatively similar to Fig. 12: the galaxy-galaxy correlation is exactly the same, and the galaxy-convergence power spectrum is simply a scaled version of the corresponding curve in Fig. 12. The major difference comes from $C_{l}^{\kappa}$ : as the $\mathrm{CMB}$ photons travel a longer path than photons from source galaxies, the convergence-convergence power spectrum is higher for the CMB lensing convergence.

On large scales $(l \lesssim 100)$, the covariance matrix is dominated by the cosmic variance terms: $1 / n_{L} \ll C_{l}^{h}$ and $N_{l}^{\kappa} \ll C_{l}^{\kappa}$. The cross-correlation coefficients are small, of order $10 \%$ : the maximum values are $0.12,0.11$, and 0.10 for $z_{L}=0.3,0.5$, and 0.8 , respectively. Therefore, we can again use Eq. (37) for estimating the variance, and find $l_{\min }$ 


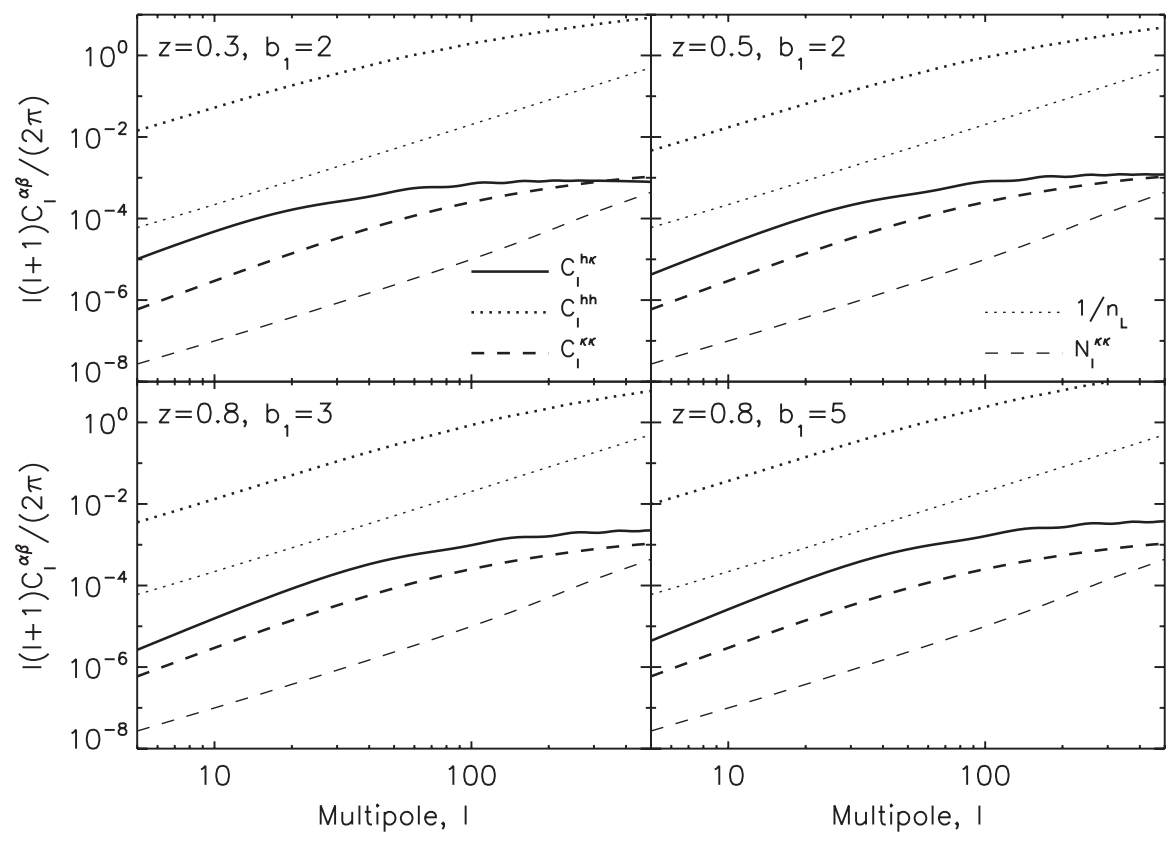

FIG. 18. Angular power spectra of the galaxy-galaxy correlation $C_{l}^{h}$ (thick dotted lines), the galaxy-convergence cross correlation $C_{l}^{h \kappa}$ (thick solid lines), and the convergence-convergence correlation $C_{l}^{\kappa}$ (thick dashed lines) for the Gaussian initial condition $\left(f_{\mathrm{NL}}=\right.$ $0)$. The four panels show the same populations of galaxies and clusters of galaxies as in Fig. 15. We also show the galaxy shot noise $1 / n_{L}$ (thin dotted lines) as well as the lens reconstruction noise $N_{l}^{\kappa}$ (think dashed lines) for $N_{L}=10^{6}$ and $N_{l}^{\kappa} \simeq 6 \times 10^{-8} \mathrm{sr}^{-1}$ (for multipoles much smaller than that corresponds to the beam size of $4^{\prime}$ ). We find $1 / n_{L} \ll C_{l}^{h}$ and $N_{l}^{\kappa} \ll C_{l}^{\kappa}$ for $l \lesssim 100$.

[Eq. (38)] above which we can measure the galaxyconvergence cross correlation with the signal-to-noise ratio greater than unity. For logarithmic bins of $\Delta l / l=0.23$, we find $l_{\min }=12.2,13.5$, and 15.8 for $z_{L}=0.3,0.5$, and 0.8 , respectively. Comparing to the results in $\mathrm{Sec}$ III C, $l_{\min }$ is slightly bigger, as $C_{l}^{\kappa}$ (which contributes to the uncertainty) increases more rapidly than $C_{l}^{h \kappa}$ (the signal we are after) would as the source redshift increases from $z_{S}$ to $z_{*}$.

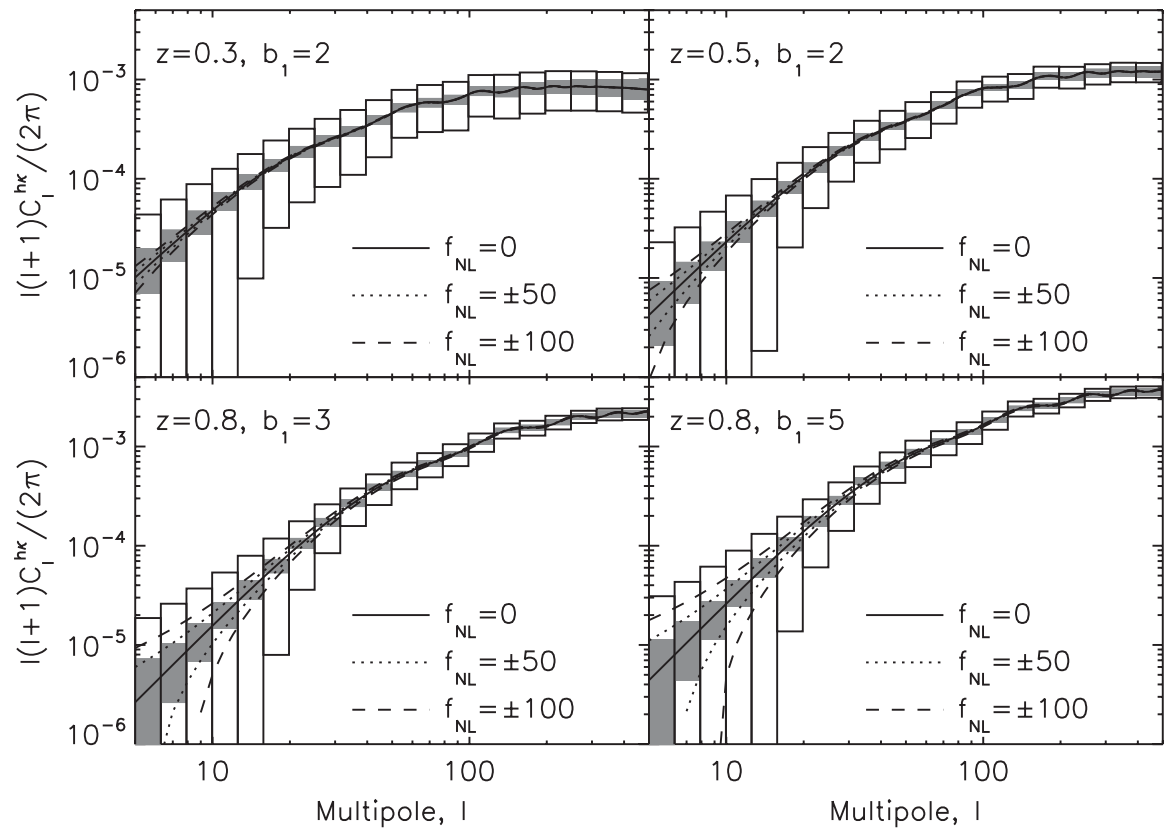

FIG. 19. Same as Fig. 15, but with 1-sigma uncertainty due to the shape noise of source galaxies [filled box, Eq. (29)] and full error budget [empty box, diagonal of Eq. (27)] including the cosmic variance. We use the multipole bins of size $\Delta l=0.23 l$. For uncertainty of $\mathrm{CMB}$ lensing reconstruction, we assume the nearly-perfect reference experiment of $\mathrm{Hu}$ and Okamoto [58]: white detector noise $\Delta_{T}=\Delta_{P} / \sqrt{2}=1 \mu \mathrm{K}$ arcmin, and FWHM of the beam $\sigma=4^{\prime}$. 


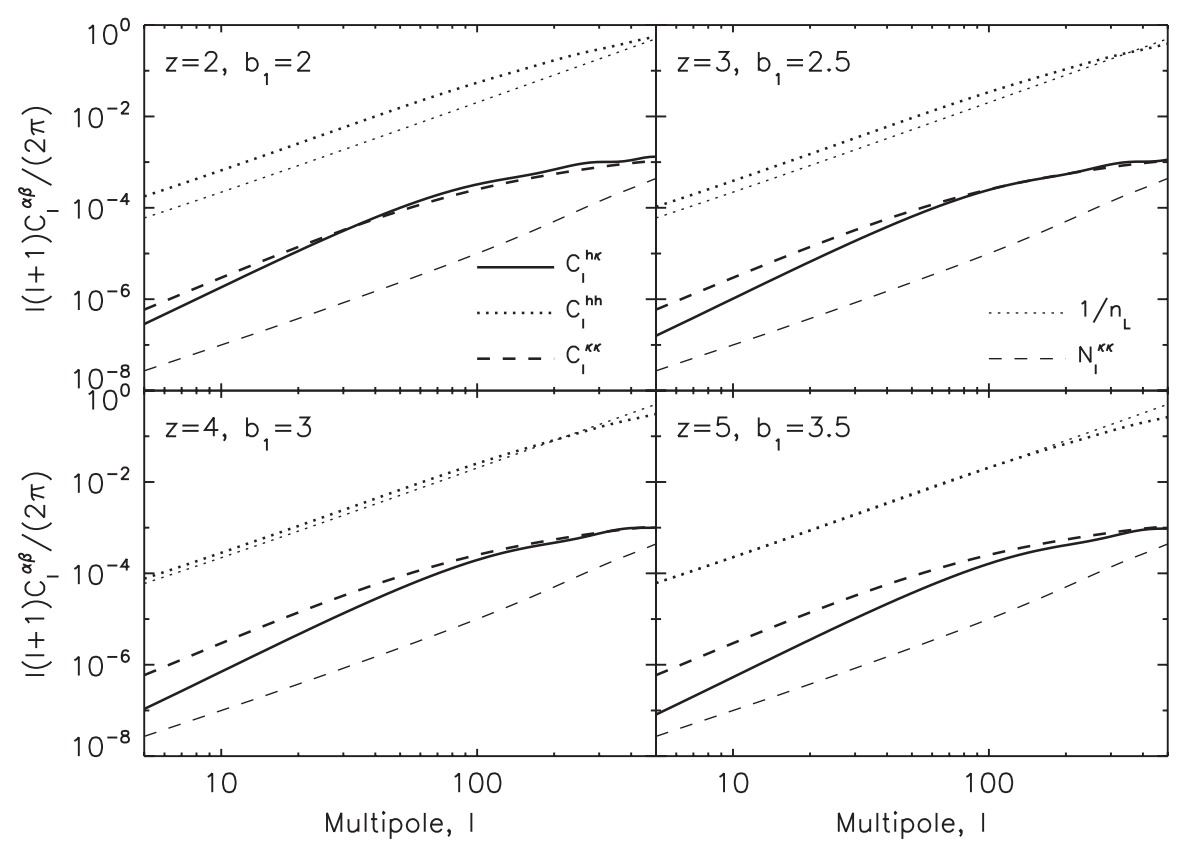

FIG. 20. Same as Fig. 18, but for the high redshift lens galaxies shown in Fig. 16. For these populations (and with $N_{L}=10^{6}$ ), the shot noise is about the same as the galaxy power spectrum, i.e., $C_{l}^{h} \simeq 1 / n_{L}$.

Figure 19 shows the expected 1- $\sigma$ uncertainties of the angular power spectrum of the galaxy-CMB lensing cross correlation, on top of the predicted Gaussian/non-Gaussian signals with five different values of non-Gaussianity parameters: $f_{\mathrm{NL}}=0, \pm 50, \pm 100$. We also show the $1-\sigma$ uncertainties without the cosmic variance due to the cosmic shear. Once again, it would be difficult to measure the effects of $f_{\mathrm{NL}}$ from a single lens redshift, but combining many slices would help measure $f_{\mathrm{NL}}$ from the galaxyCMB lensing cross correlation.

What about using even higher- $z$ lens galaxies? As shown in Fig. 20, for higher- $z$ populations (with $z_{L}=2-5$ ) the galaxy-galaxy power spectra are about the same as the shot noise levels. This is true only for the assumed number of lenses $N_{L}=10^{6}$ (over the full-sky), which is somewhat arbitrary. Increasing $N_{L}$ will help reduce the noise, but only

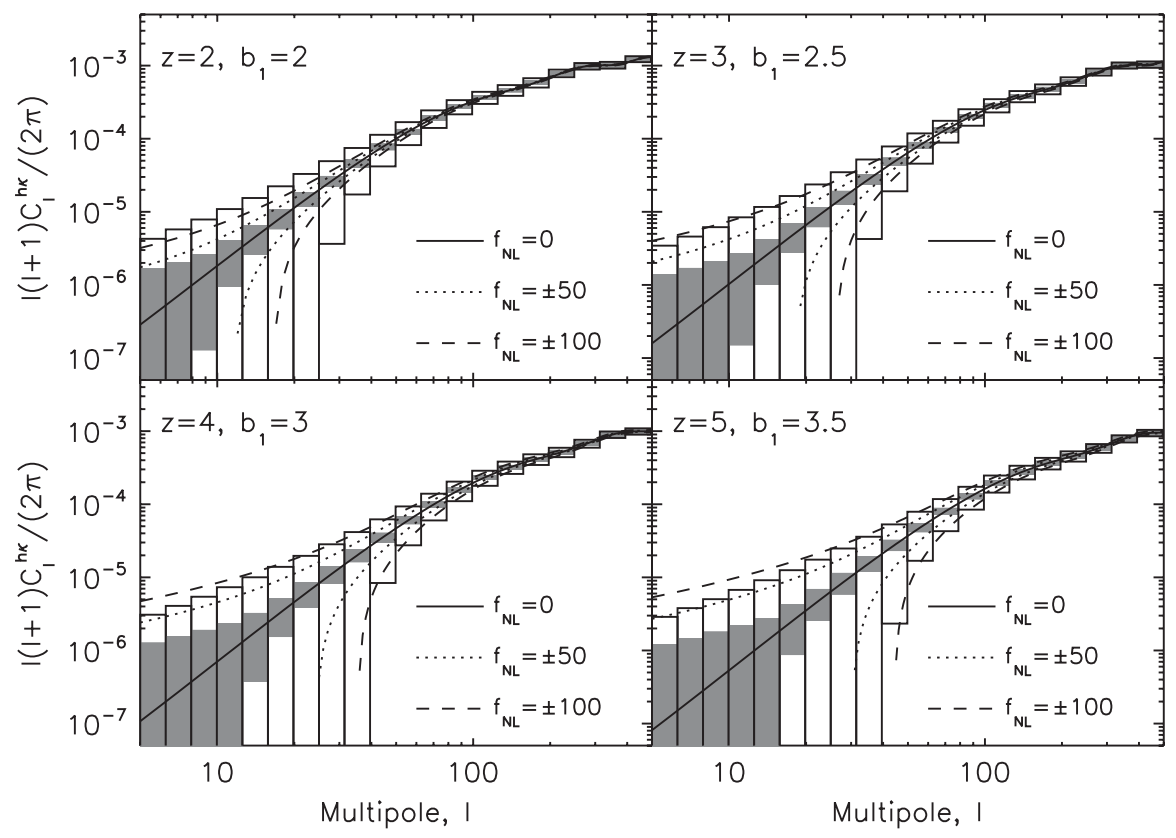

FIG. 21. Same as Fig. 19, but for the high redshift lens galaxies shown in Fig. 16. 
up to a factor of $\sqrt{2}$. For populations with $C_{l}^{h} \simeq 1 / n_{L}$, we can approximate the variance as

$$
\operatorname{Var}\left(C_{l}^{h \kappa}\right)=\frac{\left(C_{l}^{h}+1 / n_{L}\right) C_{l}^{\kappa}}{(2 l+1) \Delta l f_{\text {sky }}} \simeq \frac{2 C_{l}^{h} C_{l}^{\kappa}}{(2 l+1) \Delta l f_{\text {sky }}} .
$$

Thus, we find $C_{l}^{h \kappa} / \sqrt{\operatorname{Var}\left(C_{l}^{h \kappa}\right)} \geqslant 1$ when

$$
l \gtrsim \frac{1}{r_{l} \sqrt{(\Delta l / l) f_{\text {sky }}} .}
$$

The maximum cross-correlation coefficients are 0.091, $0.084,0.078$, and 0.073 for $z_{L}=2,3,4$, and 5 , respectively. The estimated $l_{\max }$ is then $29\left(z_{L}=2\right), 34\left(z_{L}=3\right), 38$ $\left(z_{L}=4\right)$, and $42\left(z_{L}=5\right)$.

In Fig. 21, we compare the expected 1- $\sigma$ uncertainties with the predicted signals from high- $z$ lens galaxies with $f_{\mathrm{NL}}=0, \pm 50$, and \pm 100 . Comparing this result with that in Fig. 19, we conclude that higher- $z$ lens populations do provide a better chance of finding the effects of $f_{\mathrm{NL}}$ than lower- $z$ lenses, although we would still need to combine many lens redshift slices. In particular, using higher- $z$ lenses, we can find non-Gaussian effects at higher and higher multipoles which are easier to measure; thus, high $-z$ galaxies correlated with CMB lensing offers a yet another nice probe of the local-type primordial nonGaussianity.

\section{DISCUSSION AND CONCLUSIONS}

In this paper, we have studied the galaxy-galaxy lensing and galaxy-CMB lensing cross-correlation functions. We have focused on large scales, typically larger than $100 \mathrm{Mpc}$ at the lens redshift. While current measurements have high signal-to-noise ratios on much smaller scales, we believe that future surveys will enable detection of interesting physical effects in the large-scale, linear regime.

We derive the full covariance matrix for galaxy-galaxy lensing, including the cosmic variance due to the clustering of lenses and to cosmic shear [Eq. (18)]. We use the linear bias model to provide the halo-mass and halo-halo correlations needed for this calculation. We present results for the covariance of the mean tangential shear measurement as a function of angular separations, as well as for the harmonic space halo-convergence cross-power spectrum. Our calculations show that the errors in $\Delta \Sigma(R)$ are dominated by the cosmic variance term for $R \geqslant 50 h^{-1} \mathrm{Mpc}$ (see Fig. 6). Similarly, the errors in the halo-convergence cross-power spectra $C_{l}^{h \kappa}$ are dominated by the cosmic variance term at $l \lesssim 100$ (see Fig. 19).

For Gaussian initial conditions, we show that the baryonic effects in the matter power spectrum (often called baryon acoustic oscillations) produce a shoulder in the galaxy-galaxy lensing correlation (i.e., the mean tangential shears) $\Delta \Sigma(R)$ at $R \sim 110 h^{-1} \mathrm{Mpc}$ (see Fig. 3). This effect should be easy to measure from the next-generation lensing surveys by combining $\Delta \Sigma(R)$ from multiple lens redshift slices.
We consider the prospects of detecting primordial nonGaussianity of the local form, characterized by the $f_{\mathrm{NL}}$ parameter. We have found that the scale-dependent bias from the local-form non-Gaussianity with $f_{\mathrm{NL}}= \pm 50$ modifies $\Delta \Sigma(R)$ at the level of $10-20 \%$ at $R \sim$ $300 h^{-1} \mathrm{Mpc}$ (depending on $b_{1}$ and $z_{L}$; see Fig. 5) (see Fig. 4). The modification grows rapidly toward larger scales, in proportion to $R^{2}$. High- $z$ galaxies at, e.g., $z \gtrsim$ 2, cross correlated with CMB can be used to find the effects of $f_{\mathrm{NL}}$ in the galaxy-convergence power spectrum $C_{l}^{h \kappa}$. While the effects are probably too small to see from a single lens redshift (see Fig. 21), many slices can be combined to beat down the cosmic variance errors. Exactly how many slices are necessary, or what is the optimal strategy to measure $f_{\mathrm{NL}}$ from the galaxy-CMB lensing signal requires a more detailed study that incorporates the survey strategy for specific galaxy and lens surveys.

We emphasize that, while the two-point statistics of shear fields are not sensitive to primordial nonGaussianity, the two-point statistics correlating shear fields with density peaks (i.e., galaxies and clusters of galaxies) are sensitive due to the strong scale dependence of halo bias on large scales.

Finally, we note that one can also measure the effects of $f_{\mathrm{NL}}$ on the halo power spectrum, $C_{l}^{h}$. For example, $C_{l}^{h}$ that would be measured from LSST can be used to probe $f_{\mathrm{NL}} \sim$ 1 [67]; thus, we would expect $C_{l}^{h}$ to be more powerful than the lens cross-correlation statistics we studied here. However, a combination of the two measurements would provide useful cross-checks, as galaxy clustering and galaxy-lensing correlations are affected by very different systematics.

\section{ACKNOWLEDGMENTS}

E. K. would like to thank Erin Sheldon, Rachel Mandelbaum, and Masahiro Takada for very useful discussions. B. J. thanks the UT Austin astronomy department and IUCAA for their hospitality while part of this work was carried out, and Gary Bernstein, Sarah Bridle, and Ishaana Monet for stimulating discussions. This material is based in part upon work supported by the Texas Advanced Research Program under Grant No. 0036580005-2006, by NASA Grant Nos. NNX08AM29G and NNX08AL43G, and by NSF Grant Nos. AST-0807649 and PHY-0758153. E. K. acknowledges support from the Alfred P. Sloan Foundation. D. J. acknowledges support from the University of Texas at Austin. B. J. is partially supported by NSF Grant No. AST-0908027.

\section{APPENDIX A: DERIVATION OF THE MEAN TANGENTIAL SHEAR}

One may write down the observed tangential shears at a given distance from a lens halo $\boldsymbol{\theta}$ averaged over $N_{L}$ lens halos as 


$$
\gamma_{t}^{h}(\boldsymbol{\theta})=\frac{1}{N_{L}} \int d^{2} \hat{\mathbf{n}}\left[\sum_{i}^{N_{L}} \delta_{D}\left(\hat{\mathbf{n}}-\hat{\mathbf{n}}_{i}\right)\right] \gamma_{t}(\hat{\mathbf{n}}+\boldsymbol{\theta})
$$

where $\delta_{D}$ is the delta function, and $i$ denotes the location of lens halos. Note that we have not azimuthally averaged the tangential shears yet. The ensemble average of $\gamma_{t}^{h}$ yields the number-weighted average of the tangential shear:

$$
\left\langle\gamma_{t}^{h}\right\rangle(\boldsymbol{\theta})=\frac{1}{N_{L}} \int d^{2} \hat{\mathbf{n}}\left\langle n_{L}(\hat{\mathbf{n}}) \gamma_{t}(\hat{\mathbf{n}}+\boldsymbol{\theta})\right\rangle,
$$

where $n_{L}(\hat{\mathbf{n}})$ is the surface number density of lens halos at a given location on the sky $\hat{\mathbf{n}}$. Expanding it into the perturbation $n_{L}(\hat{\mathbf{n}})=\bar{n}_{L}\left[1+\delta_{h}(\hat{\mathbf{n}})\right]$, we obtain

$$
\left\langle\gamma_{t}^{h}\right\rangle(\boldsymbol{\theta})=\frac{1}{f_{\text {sky }}} \int \frac{d^{2} \hat{\mathbf{n}}}{4 \pi}\left\langle\delta_{h}(\hat{\mathbf{n}}) \gamma_{t}(\hat{\mathbf{n}}+\boldsymbol{\theta})\right\rangle,
$$

where $f_{\text {sky }} \equiv N_{L} /\left(4 \pi \bar{n}_{L}\right)$ is a fraction of sky covered by the observation. From statistical isotropy of the Universe, $\left\langle\delta_{h}(\hat{\mathbf{n}}) \gamma_{t}(\hat{\mathbf{n}}+\boldsymbol{\theta})\right\rangle$ does not depend on $\hat{\mathbf{n}}$, and thus the integral over $\hat{\mathbf{n}}$ simply gives $4 \pi f_{\text {sky }}$. Expanding $\delta_{h}$ and $\gamma_{t}$ in Fourier space, we obtain

$$
\begin{aligned}
\left\langle\gamma_{t}^{h}\right\rangle(\boldsymbol{\theta})= & -\int \frac{d^{2} \boldsymbol{l}}{(2 \pi)^{2}} \frac{d^{2} \boldsymbol{l} \boldsymbol{}}{(2 \pi)^{2}} e^{i \boldsymbol{l} \cdot \hat{\mathbf{n}}} e^{i \boldsymbol{l} \cdot(\hat{\mathbf{n}}+\boldsymbol{\theta}) \prime} \\
& \times \cos [2(\phi-\varphi)]\left\langle\delta_{h}(\boldsymbol{l}) \kappa(\boldsymbol{l})\right\rangle \\
= & -\int \frac{d^{2} \boldsymbol{l}}{(2 \pi)^{2}} C_{l}^{h \kappa} \cos [2(\phi-\varphi)] e^{-i \boldsymbol{l} \cdot \boldsymbol{\theta}}, \boldsymbol{l}
\end{aligned}
$$

where we have used $\left\langle\delta_{h}(\boldsymbol{l}) \kappa(\boldsymbol{l})\right\rangle=(2 \pi)^{2} C_{l}^{h \kappa} \delta_{D}(\boldsymbol{l}+\boldsymbol{l})$ II. Finally, we take the azimuthal average of $\left\langle\gamma_{t}^{h}\right\rangle(\boldsymbol{\theta})$ to find the averaged mean tangential shear:

$$
\begin{aligned}
\left\langle\bar{\gamma}_{t}^{h}\right\rangle(\theta)= & \int_{0}^{2 \pi} \frac{d \phi}{2 \pi}\left\langle\gamma_{t}^{h}\right\rangle(\boldsymbol{\theta}) \\
= & -\int \frac{d^{2} \boldsymbol{l}}{(2 \pi)^{2}} C_{l}^{h \kappa} \int_{0}^{2 \pi} \frac{d \phi}{2 \pi} \cos [2(\phi-\varphi)] \\
& \times e^{-i l \theta \cos (\phi-\varphi)} \\
= & \int \frac{d^{2} \boldsymbol{l}}{(2 \pi)^{2}} C_{l}^{h \kappa} J_{2}(l \theta)=\int \frac{l d l}{2 \pi} C_{l}^{h \kappa} J_{2}(l \theta) .
\end{aligned}
$$

This completes the derivation of Eq. (11).

\section{APPENDIX B: DERIVATION OF THE COVARIANCE MATRIX OF THE MEAN TANGENTIAL SHEAR}

To compute the covariance matrix of the tangential shears (not yet azimuthally averaged), we first compute

$$
\begin{aligned}
\left\langle\gamma_{t}^{h}(\boldsymbol{\theta}) \gamma_{t}^{h}\left(\boldsymbol{\theta}^{\prime}\right)\right\rangle= & \frac{1}{N_{L}^{2}} \sum_{i j}^{N_{L}} \int d^{2} \hat{\mathbf{n}} \int d^{2} \hat{\mathbf{n}}^{\prime}\left\langle\delta_{D}\left(\hat{\mathbf{n}}-\hat{\mathbf{n}}_{i}\right)\right. \\
& \left.\times \delta_{D}\left(\hat{\mathbf{n}}^{\prime}-\hat{\mathbf{n}}_{j}\right) \gamma_{t}(\hat{\mathbf{n}}+\boldsymbol{\theta}) \gamma_{t}\left(\hat{\mathbf{n}}^{\prime}+\boldsymbol{\theta}^{\prime}\right)\right\rangle \\
= & \frac{1}{N_{L}^{2}} \int d^{2} \hat{\mathbf{n}} \int d^{2} \hat{\mathbf{n}}^{\prime}\left[\delta_{D}\left(\hat{\mathbf{n}}-\hat{\mathbf{n}}^{\prime}\right)\right. \\
& \times\left\langle n_{L}(\hat{\mathbf{n}}) \gamma_{t}(\hat{\mathbf{n}}+\boldsymbol{\theta}) \gamma_{t}\left(\hat{\mathbf{n}}^{\prime}+\boldsymbol{\theta}^{\prime}\right)\right\rangle \\
& \left.+\left\langle n_{L}(\hat{\mathbf{n}}) n_{L}\left(\hat{\mathbf{n}}^{\prime}\right) \gamma_{t}(\hat{\mathbf{n}}+\boldsymbol{\theta}) \gamma_{t}\left(\hat{\mathbf{n}}^{\prime}+\boldsymbol{\theta}^{\prime}\right)\right\rangle\right] .
\end{aligned}
$$

Here, the first term in the square bracket correlates two $\gamma_{t}$ 's measured relative to the same lens halo (1-halo term), and the second correlates two $\gamma_{t}$ 's relative to two lens halos (2halo term). Again expanding $n_{L}$ into the perturbation $n_{L}(\hat{\mathbf{n}})=\bar{n}_{L}\left[1+\delta_{h}(\hat{\mathbf{n}})\right]$, we obtain

$$
\begin{aligned}
\left\langle\gamma_{t}(\boldsymbol{\theta}) \gamma_{t}\left(\boldsymbol{\theta}^{\prime}\right)\right\rangle= & \frac{1}{f_{\text {sky }}} \frac{1}{N_{L}} \int \frac{d^{2} \hat{\mathbf{n}}}{4 \pi}\left\langle\gamma_{t}(\hat{\mathbf{n}}+\boldsymbol{\theta}) \gamma_{t}\left(\hat{\mathbf{n}}+\boldsymbol{\theta}^{\prime}\right)\right\rangle \\
& +\frac{1}{f_{\text {sky }}^{2}} \int \frac{d^{2} \hat{\mathbf{n}}}{4 \pi} \int \frac{d^{2} \hat{\mathbf{n}}^{\prime}}{4 \pi} \\
& \times\left[\left\langle\gamma_{t}(\hat{\mathbf{n}}+\boldsymbol{\theta}) \gamma_{t}\left(\hat{\mathbf{n}}^{\prime}+\boldsymbol{\theta}^{\prime}\right)\right\rangle\right. \\
& \left.+\left\langle\delta_{h}(\hat{\mathbf{n}}) \delta_{h}\left(\hat{\mathbf{n}}^{\prime}\right) \gamma_{t}(\hat{\mathbf{n}}+\boldsymbol{\theta}) \gamma_{t}\left(\hat{\mathbf{n}}^{\prime}+\boldsymbol{\theta}^{\prime}\right)\right\rangle\right] .
\end{aligned}
$$

Here, we assume that $\delta_{h}$ and $\gamma_{t}$ obey Gaussian statistics, i.e., $\left\langle\delta_{h} \gamma_{t} \gamma_{t}\right\rangle=0$. This approximation is justified even in the presence of primordial non-Gaussianity, as nonGaussianity is weak, and this approximation only affects the size of error bars. Let us evaluate each term. With $\gamma_{t}$ expanded in Fourier space, the first term (1-halo term) becomes

$$
\begin{aligned}
& \frac{1}{N_{L}} \frac{1}{f_{\text {sky }}} \int \frac{d^{2} \hat{\mathbf{n}}}{4 \pi}\left\langle\gamma_{t}(\hat{\mathbf{n}}+\boldsymbol{\theta}) \gamma_{t}\left(\hat{\mathbf{n}}+\boldsymbol{\theta}^{\prime}\right)\right\rangle \\
& =\frac{1}{N_{L}} \int \frac{d^{2} \boldsymbol{l}}{(2 \pi)^{2}} C_{l}^{\kappa} \cos [2(\phi-\varphi)] \cos \left[2\left(\phi^{\prime}-\varphi\right)\right] \\
& \quad \times e^{i l \cdot\left(\boldsymbol{\theta}-\boldsymbol{\theta}^{\prime}\right)}+\frac{\sigma_{\gamma}^{2}}{N_{L} n_{S}} \delta_{D}\left(\boldsymbol{\theta}-\boldsymbol{\theta}^{\prime}\right),
\end{aligned}
$$

where $\sigma_{\gamma}$ is the root mean square shape noise (dimensionless), and $n_{S}$ is the surface density of source (background) galaxies that are available for the shear measurement at a given location. By azimuthally averaging $\gamma_{t}$, we find

$$
\begin{aligned}
& \frac{1}{N_{L}} \int_{0}^{2 \pi} \frac{d \phi}{2 \pi} \int_{0}^{2 \pi} \frac{d \phi^{\prime}}{2 \pi}\left\langle\gamma_{t}(\hat{\mathbf{n}}+\boldsymbol{\theta}) \gamma_{t}\left(\hat{\mathbf{n}}+\boldsymbol{\theta}^{\prime}\right)\right\rangle \\
& \quad=\frac{1}{N_{L}} \int \frac{d^{2} \boldsymbol{l}}{(2 \pi)^{2}} C_{l}^{\kappa} J_{2}(l \theta) J_{2}\left(l \theta^{\prime}\right)+\frac{\sigma_{\gamma}^{2}}{N_{L} n_{S}} \frac{\delta_{D}\left(\theta-\theta^{\prime}\right)}{2 \pi \theta} .
\end{aligned}
$$

Here, $C_{l}^{\kappa}$ is the angular power spectrum of $\kappa(l)$. 
As for the second term (2-halo term), the first of the second term vanishes, as $\int d^{2} \hat{\mathbf{n}} \gamma_{t}(\hat{\mathbf{n}}+\boldsymbol{\theta})=0$. The remaining nonvanishing term gives

$$
\begin{aligned}
& \frac{1}{f_{\text {sky }}^{2}} \int \frac{d^{2} \hat{\mathbf{n}}}{4 \pi} \int \frac{d^{2} \hat{\mathbf{n}}^{\prime}}{4 \pi}\left[\left\langle\delta_{h}(\hat{\mathbf{n}}) \gamma_{t}(\hat{\mathbf{n}}+\boldsymbol{\theta})\right\rangle\left\langle\delta_{h}\left(\hat{\mathbf{n}}^{\prime}\right) \gamma_{t}\left(\hat{\mathbf{n}}^{\prime}+\boldsymbol{\theta}^{\prime}\right)\right\rangle+\left\langle\delta_{h}(\hat{\mathbf{n}}) \gamma_{t}\left(\hat{\mathbf{n}}^{\prime}+\boldsymbol{\theta}^{\prime}\right)\right\rangle\left\langle\delta_{h}\left(\hat{\mathbf{n}}^{\prime}\right) \gamma_{t}(\hat{\mathbf{n}}+\boldsymbol{\theta})\right\rangle\right. \\
& \left.\quad+\left\langle\delta_{h}(\hat{\mathbf{n}}) \delta_{h}\left(\hat{\mathbf{n}}^{\prime}\right)\right\rangle\left\langle\gamma_{t}(\hat{\mathbf{n}}+\boldsymbol{\theta}) \gamma_{t}\left(\hat{\mathbf{n}}^{\prime}+\boldsymbol{\theta}^{\prime}\right)\right\rangle\right] \\
& =\left\langle\gamma_{t}^{h}(\boldsymbol{\theta})\right\rangle\left\langle\gamma_{t}^{h}\left(\boldsymbol{\theta}^{\prime}\right)\right\rangle+\frac{1}{4 \pi f_{\mathrm{sky}}} \int \frac{d^{2} \boldsymbol{l}}{(2 \pi)^{2}} \cos [2(\phi-\varphi)] \cos \left[2\left(\phi^{\prime}-\varphi\right)\right] e^{i l \cdot\left(\boldsymbol{\theta}-\boldsymbol{\theta}^{\prime}\right)}\left[\left(C_{l}^{h \kappa}\right)^{2}+C_{l}^{h}\left(C_{l}^{\kappa}+\frac{\sigma_{\gamma}^{2}}{n_{S}}\right)\right] .
\end{aligned}
$$

Here, $C_{l}^{h}$ is the angular power spectrum of $\delta_{h}(l)$. By azimuthally averaging $\gamma_{t}$ in the above equation, we find

$$
\begin{gathered}
\left\langle\bar{\gamma}_{t}^{h}(\theta)\right\rangle\left\langle\bar{\gamma}_{t}^{h}\left(\theta^{\prime}\right)\right\rangle+\frac{1}{4 \pi f_{\text {sky }}} \int \frac{d^{2} l}{(2 \pi)^{2}} J_{2}(l \theta) J_{2}\left(l \theta^{\prime}\right) \\
\times\left[\left(C_{l}^{h \kappa}\right)^{2}+C_{l}^{h}\left(C_{l}^{\kappa}+\frac{\sigma_{\gamma}^{2}}{n_{S}}\right)\right]
\end{gathered}
$$

where we have used the identity

$$
\frac{\delta_{D}\left(\theta-\theta^{\prime}\right)}{2 \pi \theta}=\int \frac{l d l}{2 \pi} J_{2}(l \theta) J_{2}\left(l \theta^{\prime}\right) .
$$

Collecting both the 1-halo and 2-halo terms, we finally obtain the covariance matrix of the azimuthally-averaged mean tangential shear:

$$
\begin{aligned}
& \left\langle\bar{\gamma}_{t}^{h}(\theta) \bar{\gamma}_{t}^{h}\left(\theta^{\prime}\right)\right\rangle-\left\langle\bar{\gamma}_{t}^{h}(\theta)\right\rangle\left\langle\bar{\gamma}_{t}^{h}\left(\theta^{\prime}\right)\right\rangle \\
& =\frac{1}{4 \pi f_{\text {sky }}} \int \frac{l d l}{2 \pi} J_{2}(l \theta) J_{2}\left(l \theta^{\prime}\right) \\
& \quad \times\left[\left(C_{l}^{h \kappa}\right)^{2}+\left(C_{l}^{h}+\frac{1}{n_{L}}\right)\left(C_{l}^{\kappa}+\frac{\sigma_{\gamma}^{2}}{n_{S}}\right)\right] .
\end{aligned}
$$

This completes the derivation of Eq. (18).

\section{APPENDIX C: ON THE ACCURACY OF LIMBER'S APPROXIMATION}

Throughout this paper we have repeatedly used Limber's approximation in order to relate the angular correlation function to the corresponding three-dimensional power spectrum. In general, Limber's approximation is known to be accurate only for small angular scales, and only for the quantities which are integrated over a broad range of redshift.

However, the situations we have considered in this paper sometimes violate both of the conditions above: 1) We correlate the convergence field with galaxies within a very thin redshift slice, and 2) the non-Gaussianity signal we study in this paper appears only on very large scales.

Then, how accurate is Limber's approximation in this case? In this appendix, we shall study in detail the validity and limitation of Limber's approximation, by comparing the main results of the paper to the result of exact calculations.
Consider a quantity $x_{i}(\hat{\mathbf{n}})$, which is projected on the sky. Here, $\hat{\mathbf{n}}$ is the unit vector pointing toward a given direction on the sky. This quantity is related to the three-dimensional quantity $s_{i}(\mathbf{r} ; z)$ by a projection kernel $W_{i}(z)$ as

$$
x_{i}(\hat{\mathbf{n}})=\int d z W_{i}(z) s_{i}\left[d_{A}(z) \hat{\mathbf{n}} ; z\right] .
$$

Throughout this appendix, we use $d_{A}(z)$ to denote $d_{A}(0 ; z)$.

Fourier transforming $s_{i}(\mathbf{r})$, one obtains

$$
\begin{aligned}
\left.s_{i}\left[d_{A}(z) \hat{\mathbf{n}} ; z\right)\right]= & \int \frac{d^{3} k}{(2 \pi)^{3}} s_{i}(\mathbf{k}, z) e^{i \mathbf{k} \cdot \hat{\mathbf{n}} d_{A}(z)} \\
= & 4 \pi \sum_{l, m} i^{l} \int \frac{d^{3} k}{(2 \pi)^{3}} s_{i}(\mathbf{k}, z) j_{l}\left[k d_{A}(z)\right] \\
& \times Y_{l m}^{*}(\hat{\mathbf{k}}) Y_{l m}(\hat{\mathbf{n}}) .
\end{aligned}
$$

In the third line, we have used Rayleigh's formula:

$$
e^{i \mathbf{k} \cdot \hat{\mathbf{n}} r}=4 \pi \sum_{l, m} i^{l} j_{l}(k r) Y_{l m}^{*}(\hat{\mathbf{k}}) Y_{l m}(\hat{\mathbf{n}}) .
$$

By using Eq. (C2), we rewrite Eq. (C1) as

$$
\begin{aligned}
x_{i}(\hat{\mathbf{n}})= & 4 \pi \sum_{l, m} i^{l} \int d z W_{i}(z) \\
& \times \int \frac{d^{3} k}{(2 \pi)^{3}} s_{i}(\mathbf{k}, z) j_{l}\left[k d_{A}(z)\right] Y_{l m}^{*}(\hat{\mathbf{k}}) Y_{l m}(\hat{\mathbf{n}}) .
\end{aligned}
$$

Therefore, the coefficients of the spherical harmonics decomposition of $x_{i}(\hat{\mathbf{n}}), a_{l m}^{x_{i}}$, becomes

$$
a_{l m}^{x_{i}}=4 \pi i^{l} \int d z W_{i}(z) \int \frac{d^{3} k}{(2 \pi)^{3}} s_{i}(\mathbf{k}, z) j_{l}\left[k d_{A}(z)\right] Y_{l m}^{*}(\hat{\mathbf{k}}) .
$$

We calculate the angular power spectrum $C_{l}^{x_{i} x_{j}}$ by taking an ensemble average of $\left\langle a_{l m}^{x_{i}} a_{l m}^{x_{j}{ }^{*}}\right\rangle$ as

$$
\begin{aligned}
C_{l}^{x_{i} x_{j}} \equiv & \left\langle a_{l m}^{x_{i}} a_{l m}^{x_{j} *}\right\rangle \\
= & (4 \pi)^{2} \int d z W_{i}(z) \int d z^{\prime} W_{j}\left(z^{\prime}\right) \int \frac{d^{3} k}{(2 \pi)^{3}} \\
& \times P^{s_{i} s_{j}}\left(\mathbf{k} ; z, z^{\prime}\right) j_{l}\left[k d_{A}(z)\right] j_{l}\left[k d_{A}\left(z^{\prime}\right)\right] Y_{l m}^{*}(\hat{\mathbf{k}}) Y_{l m}(\hat{\mathbf{k}}),
\end{aligned}
$$


where we have used the definition of the power spectrum:

$$
\left\langle s_{i}(\mathbf{k}, z) s_{j}^{*}\left(\mathbf{k}^{\prime}, z\right)\right\rangle \equiv(2 \pi)^{3} \delta\left(\mathbf{k}-\mathbf{k}^{\prime}\right) P^{s_{i} s_{j}}\left(\mathbf{k} ; z, z^{\prime}\right) .
$$

Now, by assuming statistical isotropy of the universe, we write $P^{s_{i} s_{j}}\left(\mathbf{k} ; z, z^{\prime}\right)=P^{s_{i} s_{j}}\left(k ; z, z^{\prime}\right)$, and do the angular integration of $\hat{\mathbf{k}}$ by using the orthonormality condition of spherical harmonics:

$$
\int d \hat{\mathbf{k}} Y_{l m}(\hat{\mathbf{k}}) Y_{l m}^{*}(\hat{\mathbf{k}})=1 .
$$

We then obtain the angular power spectrum given by

$$
\begin{aligned}
C_{l}^{x_{i} x_{j}}= & \int d z W_{i}(z) \int d z^{\prime} W_{j}\left(z^{\prime}\right) \\
& \times\left\{\frac{2}{\pi} \int k^{2} d k P^{s_{i} s_{j}}\left(k ; z, z^{\prime}\right) j_{l}\left[k d_{A}(z)\right] j_{l}\left[k d_{A}\left(z^{\prime}\right)\right]\right\} .
\end{aligned}
$$

This is the exact relation.

What determines the form of $W_{i}(z)$ ? For a projected galaxy distribution projected on the sky, this kernel is simply a normalized galaxy distribution function in redshift space. In this paper, we consider the delta functionlike distribution, i.e.,

$$
W_{g}(z)=\delta^{D}\left(z-z_{L}\right) .
$$

Using Eq. (C6) with the delta function kernel above yields Eq. (36):

$$
C_{l}^{h}=\frac{2}{\pi} \int d k k^{2} P_{g}\left(k, z_{L}\right) j_{l}^{2}\left[k d_{A}\left(z_{L}\right)\right]
$$

Again, this is still the exact result. As the form of $W_{g}(z)$ we have considered here (i.e., a delta function) is a sharply peaked function, we cannot use Limber's approximation given below. This is the reason why we have used the exact result for $C_{l}^{h}$.

In order to get the expression for Limber's approximation, we assume that $P^{s_{i} s_{j}}(k)$ is a slowly-varying function of $k$. Then, by using the identity

$$
\frac{2}{\pi} \int k^{2} d k j_{l}(k r) j_{l}\left(k r^{\prime}\right)=\frac{\delta^{D}\left(r-r^{\prime}\right)}{r^{2}},
$$

we approximate the $k$ integral of Eq. (C6) as

$$
\begin{aligned}
& \frac{2}{\pi} \int k^{2} d k P^{s_{i} s_{j}}(k) j_{l}(k r) j_{l}\left(k r^{\prime}\right) \\
& \approx \frac{\delta^{D}\left(r-r^{\prime}\right)}{r^{2}} P^{s_{i} s_{j}}\left(k=\frac{l+1 / 2}{r}\right) .
\end{aligned}
$$

By using this approximation, we finally get

$$
C_{l}^{x_{i} x_{j}} \approx \int d z W_{i}(z) W_{j}(z) \frac{H(z)}{d_{A}^{2}(z)} P^{s_{i} s_{j}}\left(k=\frac{l+1 / 2}{r} ; z\right),
$$

which is the result known as Limber's approximation.

One important application of Limber's approximation is the statistics involving weak gravitational lensing. The lensing kernel for the convergence field $W_{\kappa}(z)$ can be calculated by integrating the lens equation:

$$
W_{\kappa}(z)=\frac{\rho_{0}}{\Sigma_{c}\left(z ; z_{S}\right) H(z)},
$$

where $\Sigma_{c}\left(z ; z_{S}\right)$ is the critical surface density defined in Eq. (16). The exact result for the galaxy-convergence angular cross-power spectrum is

$$
\begin{aligned}
C_{l}^{h \kappa}\left(z_{L}\right)= & \frac{2}{\pi} \int_{0}^{z_{S}} d z \frac{\rho_{0}}{\Sigma_{c}\left(z ; z_{S}\right) H(z)} \\
& \times \int d k k^{2} P_{h m}\left(k, z_{L}, z\right) j_{l}\left[k d_{A}\left(z_{L}\right)\right] j_{l}\left[k d_{A}(z)\right],
\end{aligned}
$$

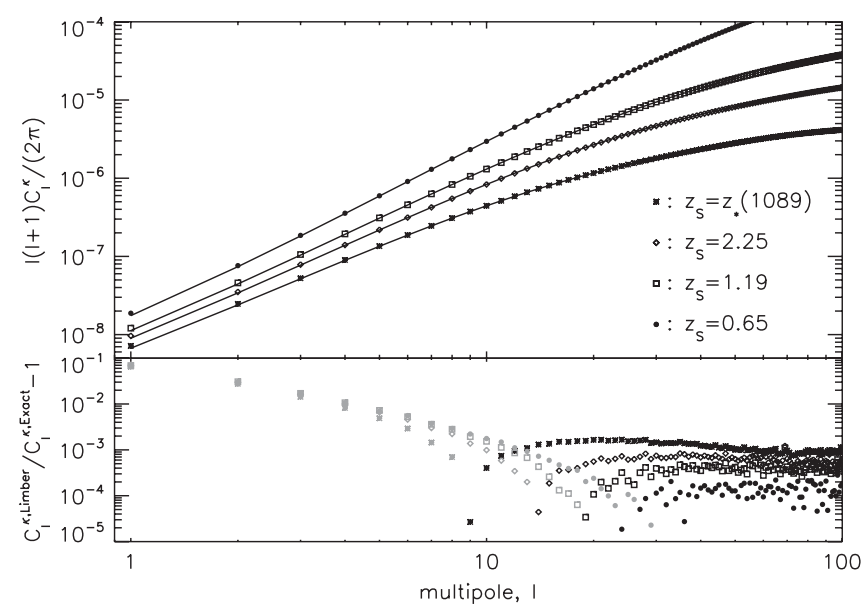

FIG. 22. Top: Convergence-convergence angular power spectrum from two different methods: the exact calculation [Eq. (C14), symbols] and Limber's approximation [Eq. (35), solid lines]. Bottom: Fractional differences between Limber's approximation and the exact integration. Symbols are the same as the top panel. Grey symbols show the absolute values of negative values.

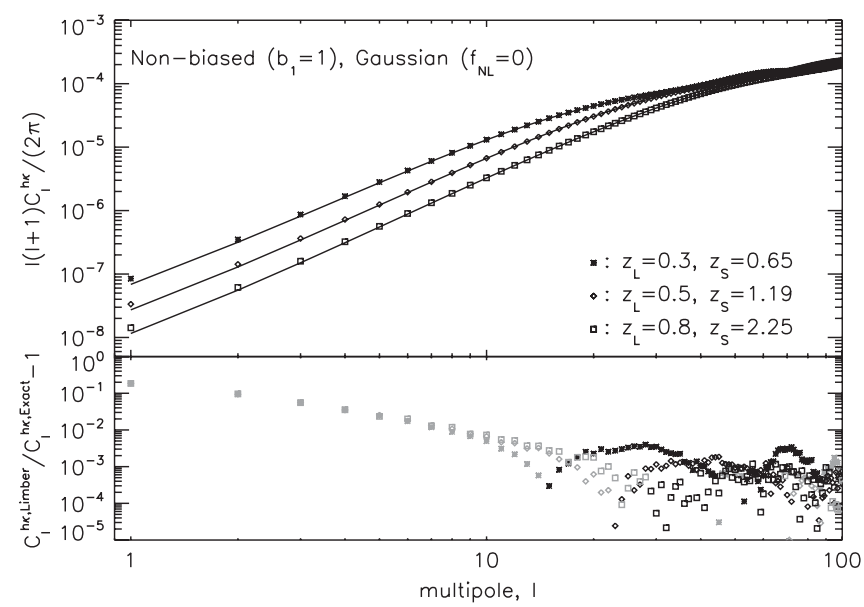

FIG. 23. Same as Fig. 22, but for the galaxy-convergence cross angular power spectrum with $f_{\mathrm{NL}}=0$ and $b_{1}=1$. 


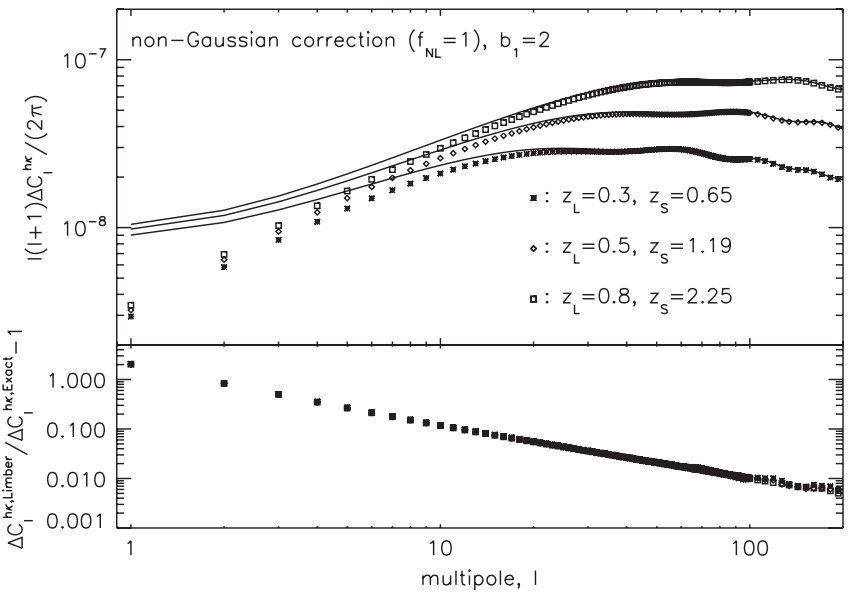

FIG. 24. Same as Fig. 22, but for the non-Gaussian correction (i.e., the term proportional to $\Delta b(k))$ to the galaxy-convergence cross angular power spectrum. We show the corrections with $f_{\mathrm{NL}}=1$ and $b_{1}=2$.

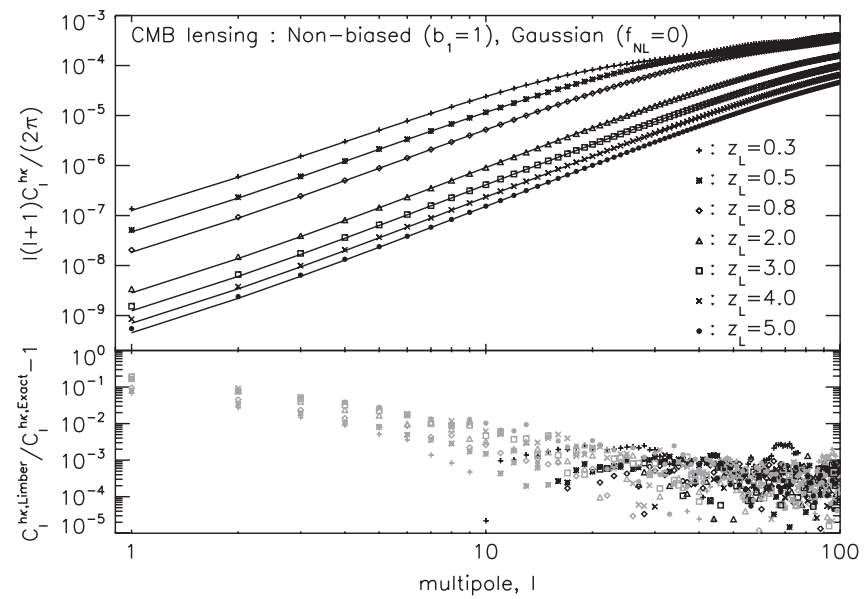

FIG. 25. Same as Fig. 23, but for the galaxy-CMB lensing.

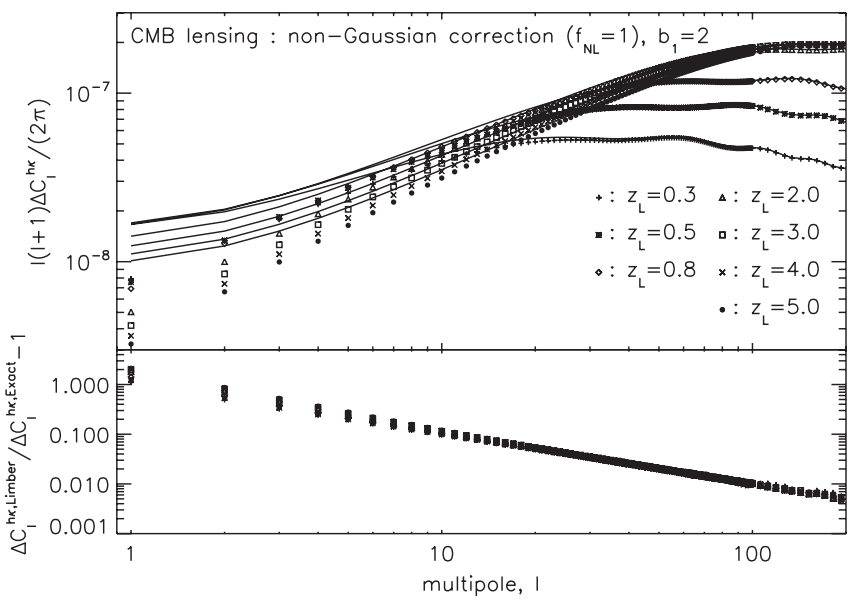

FIG. 26. Same as Fig. 24, but for the galaxy-CMB lensing.

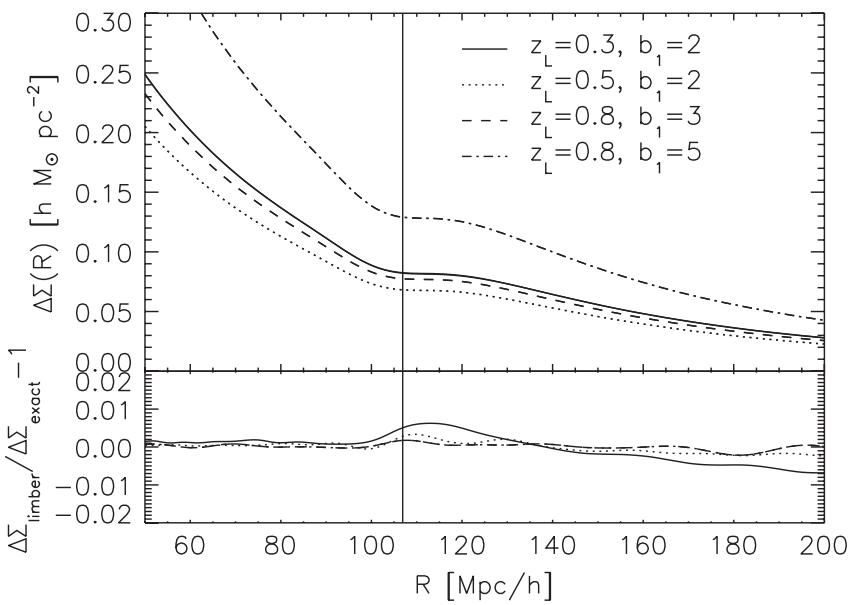

FIG. 27. Top: Same as Fig. 3, but also showing the exact result [Eq. (C13), thick lines] on top of the result from Limber's approximation [Eq. (32), thin lines]. Bottom: Fractional difference of Limber's approximation relative to the exact result.

and the exact result for the convergence-convergence angular power spectrum is

$$
\begin{aligned}
C_{l}^{\kappa}\left(z_{S}\right)= & \frac{2}{\pi} \int_{0}^{z_{S}} d z \int_{0}^{z_{S}} d z^{\prime} \frac{\rho_{0}^{2}}{\Sigma_{c}\left(z ; z_{S}\right) H(z) \Sigma_{c}\left(z^{\prime} ; z_{S}\right) H\left(z^{\prime}\right)} \\
& \times \int d k k^{2} P_{m}\left(k, z, z^{\prime}\right) j_{l}\left[k d_{A}(z)\right] j_{l}\left[k d_{A}\left(z^{\prime}\right)\right] .
\end{aligned}
$$

First, we compare the exact convergence-convergence angular power spectrum to Limber's approximation. Figure 22 shows that Limber's approximation works very well for all four source redshifts we study in the paper:

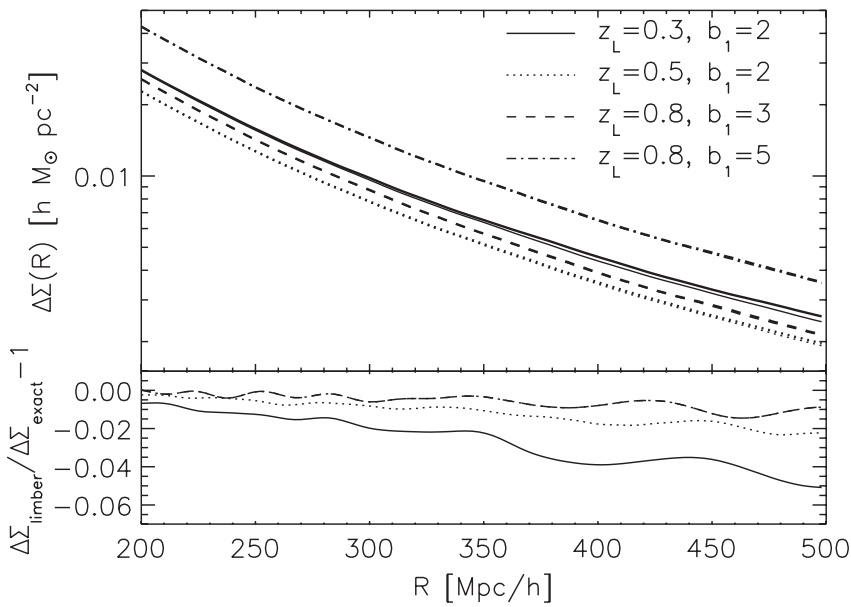

FIG. 28. Same as Fig. 27, but for larger $R$. Thick lines are the results of the exact integration, while the thin lines are Limber's approximation. The Limber approximation over predicts $\Delta \Sigma(R)$ for large $R$, but the error is at most $5 \%$ for $R<500 h^{-1} \mathrm{Mpc}$. The error is the largest for the lowest $z_{L}$, as a physical separation $R$ at a lower redshift corresponds to a larger angular separation on the sky. 


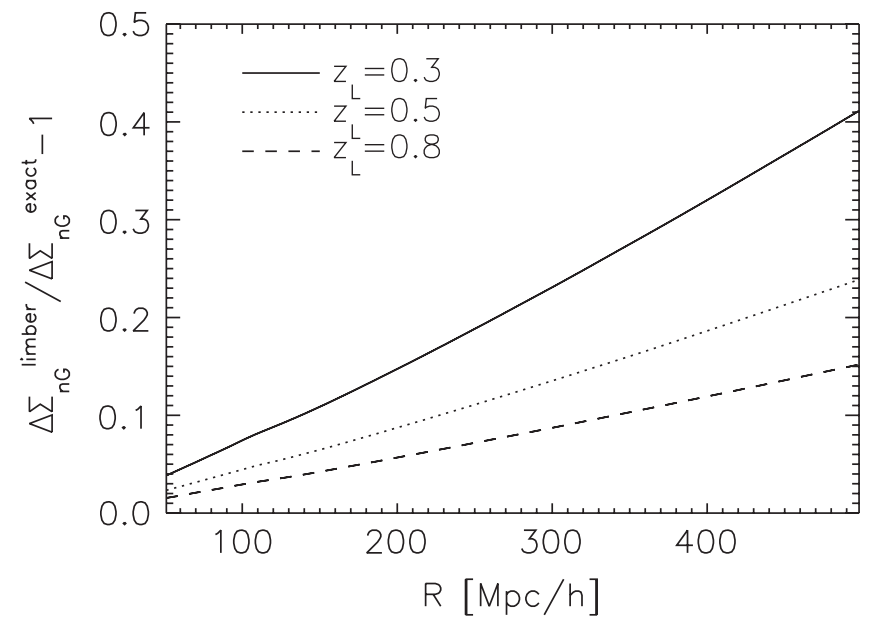

FIG. 29. Fractional differences in the non-Gaussian correction terms, $\Delta \Sigma_{\mathrm{nG}}$, from Limber's approximation and the exact integration. Using Limber's approximation, we overpredict the nonGaussian correction by $\sim 20 \%$ at $R=300 \mathrm{~h}^{-1} \mathrm{Mpc}$ for $z_{L}=0.3$.

$z_{S}=0.65,1.19,2.25$, and 1089.0. For $l>10$, the error caused by Limber's approximation is always much smaller than $1 \%$.

Then, we compare the galaxy-convergence cross angular power spectra. Figures 23 and 24 show the comparison between the exact galaxy-convergence cross-power spectrum [Eq. (C13), symbols] and their Limber approximation [Eq. (32), solid lines] for three galaxy-galaxy lensing cases we study in Sec. II: $\left(z_{L}, z_{S}\right)=(0.3,0.65),(0.5,1.19)$, and $(0.8,2.25)$.

For the Gaussian term (Fig. 23), Limber's approximation is accurate at $l>10$ with the errors less than $1 \%$. On the other hand, Limber's approximation to the non-
Gaussian correction term (Fig. 24) has a sizable error, at the level of $10 \%$, at $l \sim 10$. The error goes down to the $1 \%$ level only at $l \sim 100$. One needs to keep this in mind when comparing Limber's approximation with observations. We find that Limber's approximation underpredicts the Gaussian term at $l \leqq 20$, while it overpredicts the nonGaussian corrections at all multipoles.

The story is basically the same for the galaxy-CMB lensing cross-power spectrum. Figure 25 (Gaussian term) and Fig. 26 (non-Gaussian correction) show the comparison between the exact galaxy-convergence cross-power spectrum [Eq. (C13), solid lines] and their Limber approximation [Eq. (32), dashed lines] for seven lens redshifts we study in Sec. IV: $z_{L}=0.3,0.5,0.8,2,3,4$, and 5. Again, for small scales $l>10$ Limber's approximation works better than $1 \%$ for the Gaussian term, while it overpredicts the non-Gaussian correction at the level of $10 \%$ at $l \sim 10$ and $1 \%$ at $l \sim 100$.

What about the effect on the mean tangential shear $\Delta \Sigma(R)$ ? Figure 27 compares the Gaussian term of $\Delta \Sigma(R)$ from the exact integration and that from Limber's approximation. On the top panel of Fig. 27, we show the baryonic feature computed with Limber's approximation (thin lines, the same as those in Fig. 3) as well as that computed with the exact integration (thick lines). They are indistinguishable by eye. The bottom panel shows the fractional differences between the two. We find that Limber's approximation is better than $0.5 \%$ for $R<180 h^{-1} \mathrm{Mpc}$; thus, the baryonic feature in $\Delta \Sigma$ is not an artifact caused by Limber's approximation.

However, Limber's approximation becomes worse and worse as we go to larger $R$. Figure 28 shows $\Delta \Sigma$ on large scales. For the lens redshifts that we have studied here, the error is at most $5 \%$ for $R<500 h^{-1} \mathrm{Mpc}$, and the error is

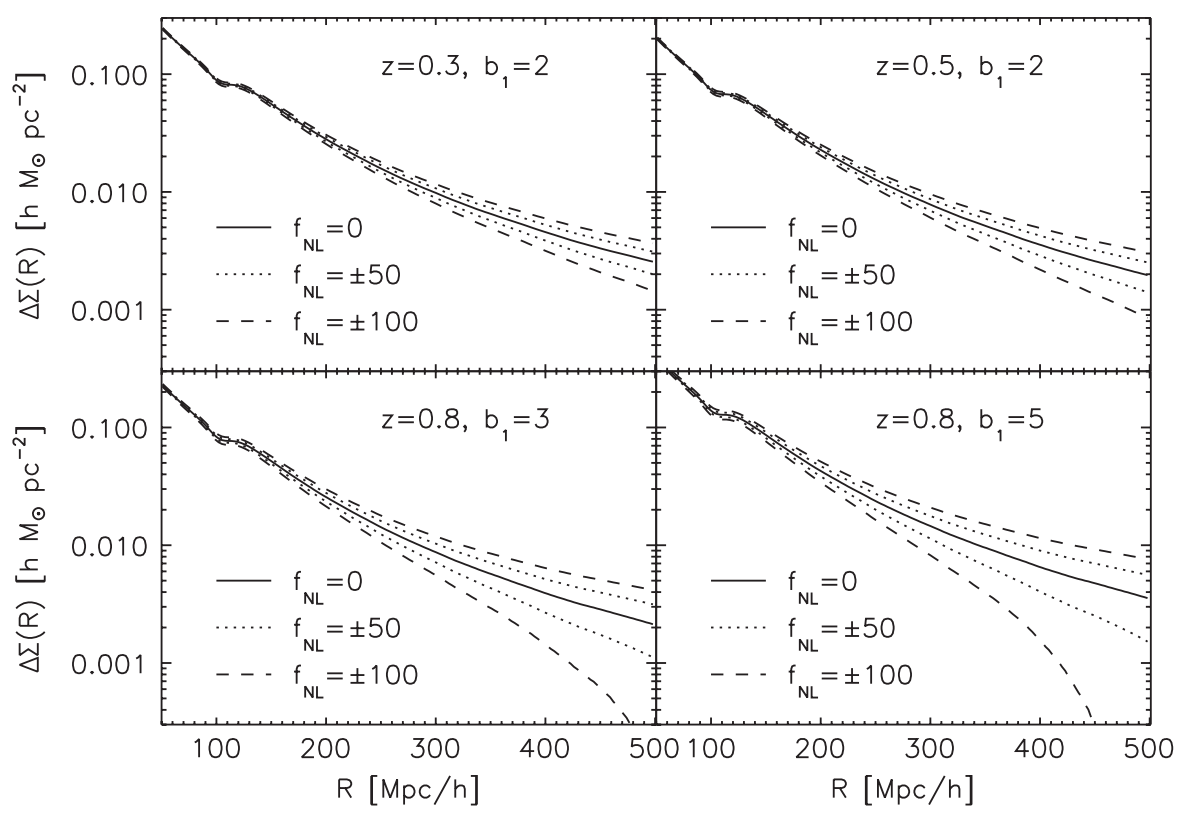

FIG. 30. Same as Fig. 4, but with the exact integration instead of Limber's approximation. 
the largest for the lowest $z_{L}$, as a given $R$ at a lower redshift corresponds to a larger angular separation on the sky.

While Limber's approximation underpredicts the Gaussian term on large scales, it overpredicts the nonGaussian correction terms. Figure 29 shows the fractional differences of the non-Gaussian correction terms, $\Delta \Sigma_{\mathrm{nG}}$, between Limber's approximation and the exact calculation as a function of separation $R$ for three lens redshifts: $z_{L}=$ $0.3,0.5$, and 0.8 . This figure shows that the error caused by Limber's approximation can be substantial on $\Delta \Sigma_{\mathrm{nG}}$.
As Limber's approximation to $\Delta \Sigma(R)$ can be quite inaccurate on very large scales, we show the exact calculations of $\Delta \Sigma(R)$ in Fig. 30. (Limber's approximation is given in Fig. 4.)

Finally, we note that the definition of the tangential shear we have used [Eq. (4)] is valid only on the flat sky (as noted in the footnote there), and thus the prediction for $\Delta \Sigma$ on very large scales probably needs to be revisited with the exact definition of the tangential shears on the full sky using the spin-2 harmonics. This is beyond of the scope of our paper.
[1] E. Komatsu and D. N. Spergel, Phys. Rev. D 63, 063002 (2001).

[2] E. Komatsu, J. Dunkley, M. R. Nolta, C. L. Bennett, B. Gold, G. Hinshaw, N. Jarosik, D. Larson, M. Limon, L. Page et al., Astrophys. J. Suppl. Ser. 180, 330 (2009).

[3] D. Babich, P. Creminelli, and M. Zaldarriaga, J. Cosmol. Astropart. Phys. 08 (2004) 009.

[4] D. S. Salopek and J.R. Bond, Phys. Rev. D 42, 3936 (1990).

[5] T. Falk, R. Rangarajan, and M. Srednicki, Astrophys. J. Lett. 403, L1 (1993).

[6] A. Gangui, F. Lucchin, S. Matarrese, and S. Mollerach, Astrophys. J. 430, 447 (1994).

[7] J. M. Maldacena, J. High Energy Phys. 05 (2003) 013.

[8] V. Acquaviva, N. Bartolo, S. Matarrese, and A. Riotto, Nucl. Phys. B667, 119 (2003).

[9] P. Creminelli and M. Zaldarriaga, Phys. Rev. D 70, 083532 (2004).

[10] D. Seery and J. E. Lidsey, J. Cosmol. Astropart. Phys. 06 (2005) 003.

[11] X. Chen, M.-x. Huang, S. Kachru, and G. Shiu, J. Cosmol. Astropart. Phys. 01 (2007) 002.

[12] C. Cheung, P. Creminelli, A. L. Fitzpatrick, J. Kaplan, and L. Senatore, J. High Energy Phys. 03 (2008) 014.

[13] N. Bartolo, E. Komatsu, S. Matarrese, and A. Riotto, Phys. Rep. 402, 103 (2004).

[14] E. Komatsu et al., arXiv:0902.4759.

[15] K. M. Smith, L. Senatore, and M. Zaldarriaga, J. Cosmol. Astropart. Phys. 09 (2009) 006.

[16] L. Verde, L. Wang, A. F. Heavens, and M. Kamionkowski, Mon. Not. R. Astron. Soc. 313, 141 (2000).

[17] L. Wang and M. Kamionkowski, Phys. Rev. D 61, 063504 (2000).

[18] E. Komatsu, B. D. Wandelt, D. N. Spergel, A. J. Banday, and K. M. Górski, Astrophys. J. 566, 19 (2002).

[19] E. Komatsu, A. Kogut, M. R. Nolta, C. L. Bennett, M. Halpern, G. Hinshaw, N. Jarosik, M. Limon, S. S. Meyer, L. Page et al., Astrophys. J. Suppl. Ser. 148, 119 (2003).

[20] E. Komatsu, Ph.D. thesis, Tohoku University, 2001, arXiv: astro-ph/0206039.

[21] T. Okamoto and W. Hu, Phys. Rev. D 66, 063008 (2002).

[22] N. Kogo and E. Komatsu, Phys. Rev. D 73, 083007 (2006).
[23] R. Scoccimarro, E. Sefusatti, and M. Zaldarriaga, Phys. Rev. D 69, 103513 (2004).

[24] E. Sefusatti and E. Komatsu, Phys. Rev. D 76, 083004 (2007).

[25] D. Jeong and E. Komatsu, Astrophys. J. 703, 1230 (2009).

[26] E. Sefusatti, Phys. Rev. D 80, 123002 (2009).

[27] F. Lucchin and S. Matarrese, Astrophys. J. 330, 535 (1988).

[28] S. Matarrese, L. Verde, and R. Jimenez, Astrophys. J. 541, 10 (2000).

[29] E. Sefusatti, C. Vale, K. Kadota, and J. Frieman, Astrophys. J. 658, 669 (2007).

[30] M. LoVerde, A. Miller, S. Shandera, and L. Verde, J. Cosmol. Astropart. Phys. 04 (2008) 014.

[31] N. Dalal, O. Doré, D. Huterer, and A. Shirokov, Phys. Rev. D 77, 123514 (2008).

[32] S. Matarrese and L. Verde, Astrophys. J. Lett. 677, L77 (2008).

[33] A. Slosar, C. Hirata, U. Seljak, S. Ho, and N. Padmanabhan, J. Cosmol. Astropart. Phys. 8 (2008) 031.

[34] N. Afshordi and A. J. Tolley, Phys. Rev. D 78, 123507 (2008).

[35] A. Taruya, K. Koyama, and T. Matsubara, Phys. Rev. D 78, 123534 (2008).

[36] V. Desjacques, U. Seljak, and I. T. Iliev, arXiv:0811.2748.

[37] A. Pillepich, C. Porciani, and O. Hahn, arXiv:0811.4176.

[38] M. Grossi, L. Verde, C. Carbone, K. Dolag, E. Branchini, F. Iannuzzi, S. Matarrese, and L. Moscardini, arXiv:0902.2013.

[39] M. Bartelmann and P. Schneider, Phys. Rep. 340, 291 (2001).

[40] J. A. Tyson, F. Valdes, J. F. Jarvis, and A. P. Mills, Jr., Astrophys. J. Lett. 281, L59 (1984).

[41] T. G. Brainerd, R. D. Blandford, and I. Smail, Astrophys. J. 466, 623 (1996).

[42] T. A. McKay, E. S. Sheldon, J. Racusin, P. Fischer, U. Seljak, A. Stebbins, D. Johnston, J. A. Frieman, N. Bahcall, J. Brinkmann et al., arXiv:astro-ph/0108013.

[43] J. Guzik and U. Seljak, Mon. Not. R. Astron. Soc. 335, 311 (2002).

[44] E. S. Sheldon, D. E. Johnston, J. A. Frieman, R. Scranton, T. A. McKay, A. J. Connolly, T. Budavári, I. Zehavi, N. A. Bahcall, J. Brinkmann et al., Astron. J. 127, 2544 (2004). 
[45] R. Mandelbaum, U. Seljak, G. Kauffmann, C. M. Hirata, and J. Brinkmann, Mon. Not. R. Astron. Soc. 368, 715 (2006).

[46] R. Mandelbaum, U. Seljak, R. J. Cool, M. Blanton, C. M. Hirata, and J. Brinkmann, Mon. Not. R. Astron. Soc. 372, 758 (2006)

[47] A. Stebbins, arXiv:astro-ph/9609149.

[48] E. S. Sheldon, D. E. Johnston, R. Scranton, B. P. Koester, T. A. McKay, H. Oyaizu, C. Cunha, M. Lima, H. Lin, J. A. Frieman et al., Astrophys. J. 703, 2217 (2009).

[49] D. E. Johnston, E. S. Sheldon, R. H. Wechsler, E. Rozo, B. P. Koester, J. A. Frieman, T. A. McKay, A. E. Evrard, M. R. Becker, and J. Annis, arXiv:0709.1159.

[50] W. Hu and B. Jain, Phys. Rev. D 70, 043009 (2004).

[51] D. N. Limber, Astrophys. J. 119, 655 (1954).

[52] N. Kaiser, Astrophys. J. 388, 272 (1992).

[53] M. Tegmark, D. J. Eisenstein, M. A. Strauss, D. H. Weinberg, M. R. Blanton, J. A. Frieman, M. Fukugita, J.E. Gunn, A. J.S. Hamilton, G. R. Knapp et al., Phys. Rev. D 74, 123507 (2006).

[54] D. Schlegel, M. White, and D. Eisenstein, arXiv: 0902.4680.
[55] H. Zhan, J. Cosmol. Astropart. Phys. 8 (2006) 008.

[56] D. J. Eisenstein and W. Hu, Astrophys. J. 496, 605 (1998).

[57] A. Lewis and A. Challinor, Phys. Rep. 429, 1 (2006).

[58] W. Hu and T. Okamoto, Astrophys. J. 574, 566 (2002).

[59] T. Okamoto and W. Hu, Phys. Rev. D 67, 083002 (2003).

[60] C. M. Hirata and U. Seljak, Phys. Rev. D 68, 083002 (2003).

[61] S. Boughn and R. Crittenden, Nature (London) 427, 45 (2004).

[62] R. Mandelbaum, C. M. Hirata, U. Seljak, J. Guzik, N. Padmanabhan, C. Blake, M. R. Blanton, R. Lupton, and J. Brinkmann, Mon. Not. R. Astron. Soc. 361, 1287 (2005).

[63] G. J. Hill, K. Gebhardt, E. Komatsu, and P. J. MacQueen, AIP Conf. Proc. 743, 224 (2004).

[64] G. J. Hill et al., ASP Conf. Ser. 399, 115 (2008).

[65] V. Acquaviva, A. Hajian, D. N. Spergel, and S. Das, Phys. Rev. D 78, 043514 (2008).

[66] L. Knox, Phys. Rev. D 52, 4307 (1995).

[67] C. Carbone, L. Verde, and S. Matarrese, Astrophys. J. Lett. 684, L1 (2008). 